\title{
Localization of a multi-dimensional quantum walk with one defect
}

\author{
Toru Fuda, Daiju Funakawa †and Akito Suzuki ${ }^{\ddagger}$
}

\begin{abstract}
In this paper, we introduce a multidimensional generalization of Kitagawa's splitstep discrete-time quantum walk, study the spectrum of its evolution operator for the case of one defect coins, and prove localization of the walk. Using a spectral mapping theorem, we can reduce the spectral analysis of the evolution operator to that of a discrete Schrödinger operator with variable coefficients, which is analyzed using the Feshbach map.
\end{abstract}

Keywords: quantum walks, localization, eigenvalues, Feshbach map

\section{Introduction}

Quantum walks (QWs) have been introduced and studied in various contexts such as quantum probability [13], quantum optics [1], quantum cellular automata, [15, 28], and quantum information [2, 8] (see [4, 21, 24, 37] for more details). Among them, motivated by Grover's quantum search algorithms [14, 35, 10], researchers have proposed several types of discrete time QWs on graphs [38, 3, 22, 20, 25, 36]. Szegedy [32] introduced a bipartite walk, which is defined on a bipartite graph, to construct a quantum search algorithm. Magniez et al [29, 30] updated the notion of bipartite walks and Segawa [33] redefined an evolution operator $U_{G}$ on the Hilbert space $\ell^{2}(D)$ of square summable functions on the set $D$ of arcs for a digraphs $G=(V, D)$. The $\mathrm{QW}$ defined by $U_{G}$ is now referred to as the Szgedy walk on $G$, which includes the Grover walk on $G$ as a special case. The Szegedy walks have a spectral mapping property from the transition probability matrix $P_{G}$ of a random walk on $G$ to the evolution $U_{G}$, which gives a useful tool for analyzing the

\footnotetext{
${ }^{*}$ Department of Mathematics, Hokkaido University, Sapporo 060-0810, Japan, E-mail: t-fuda@math.sci.hokudai.ac.jp

${ }^{\dagger}$ Department of Mathematics, Hokkaido University, Sapporo 060-0810, Japan, E-mail: funakawa@math.sci.hokudai.ac.jp

${ }^{\ddagger}$ Division of Mathematics and Physics, Faculty of Engineering, Shinshu University, Wakasato, Nagano 380-8553, Japan, E-mail: akito@shinshu-u.ac.jp
} 
spectrum of $U_{G}$ (see [33, 27, 17] for more details). An extended version of the Szegedy walk, the twisted Szegedy walk, was introduced by Higuchi et al [16] to study the spectral and asymptotic properties of the Grover walks on crystal lattices. Higuchi, Segawa, and one of the authors of this paper [18] proved the spectral mapping theorem (SMT) for more general evolution $U=S C$, where $S$ and $C$ are unitary and self-adjoint on a Hilbert space $\mathcal{H}$ and where $C$ is assumed to be of the form

$$
C=2 d^{*} d-1
$$

with a coisometry $d$ from $\mathcal{H}$ to a Hilbert space $\mathcal{K}$, i.e., $d d^{*}$ is the identity $I_{\mathcal{K}}$ on $\mathcal{K}$. Observe that the Hilbert space $\mathcal{H}$ here can be taken to be arbitrary and is no longer needed to be $\ell^{2}(D)$. Let $T=d S d^{*}$. $T$ is a self-adjoint operator on $\mathcal{K}$ and called the discriminant operator of $U$. Let $\mathcal{D}_{ \pm}=\operatorname{ker} d \cap \operatorname{ker}(S \pm 1)$. The subspace $\mathcal{D}_{\mathrm{B}}=\mathcal{D}_{+} \oplus \mathcal{D}_{-} \subset \mathcal{H}$ is called the birth eigenspace of $U$ and its orthogonal complement $\mathcal{D}_{\mathrm{I}}$ the inherited subspace of $U$ (see [17, 26]). As shown elsewhere [34], the restriction $U_{\mathrm{I}}:=\left.U\right|_{\mathcal{D}_{\mathrm{I}}}$ to the inherited subspace is unitarily equivalent to

$$
\exp (+i \arccos T) \oplus \exp (-i \arccos T)
$$

and the restriction $U_{\mathrm{B}}:=\left.U\right|_{\mathcal{D}_{\mathrm{B}}}$ to the birth eigenspace is $I_{\mathcal{D}_{+}} \oplus\left(-I_{\mathcal{D}_{-}}\right)$. Thus, the spectral analysis of $U$ is reduced to two parts: (1) the spectral analysis of $T$ and (2) the calculation of $\operatorname{dim} \mathcal{D}_{\mathrm{B}}$. This reduction leads the SMT from $T$ to $U$ (Theorem 2.1), which allows us to use it for QWs other than the Szegedy walk. As evident below, such an abstract theorem is applicable for a class of $d$-dimensional QWs, which is not the Szegedy walk on $\mathbb{Z}^{d}$. In forthcoming papers [11, 12, we will consider a unified model that includes a split-step QW introduced by Kitagawa et al [23] and traditional one-dimensional QWs [2, 13, 28] as special cases. The evolution of the walk is a unitary operator on $\ell^{2}\left(\mathbb{Z} ; \mathbb{C}^{2}\right)$ defined as $U=S_{1} C$, where

$$
\left(S_{1} \psi\right)(x)=\left(\begin{array}{c}
p \psi_{1}(x)+q \psi_{2}(x+1) \\
q^{*} \psi_{1}(x-1)-p \psi_{2}(x)
\end{array}\right), \quad x \in \mathbb{Z}, \psi \in \ell^{2}\left(\mathbb{Z} ; \mathbb{C}^{2}\right) .
$$

Taking $(p, q) \in \mathbb{R} \times \mathbb{C}$ as $p^{2}+|q|^{2}=1$ ensures $S_{1}$ is unitary and self-adjoint. $C$ is a multiplication operator by unitary matrices $C(x) \in U(2)$. If $C(x)$ is in addition hermitian and $\operatorname{dim} \operatorname{ker}(C(x)-1)=1$ for all $x \in \mathbb{Z}$, then $C$ is written as $2 d^{*} d-1$ with a coisometry $d: \ell^{2}\left(\mathbb{Z} ; \mathbb{C}^{2}\right) \rightarrow \ell^{2}(\mathbb{Z})$ (see Example 2.2). Thus the SMT is applicable.

Models In this paper, we consider a multi-dimensional generalization of the aforementioned model, which is a $2 d$-state QW on $\mathbb{Z}^{d}$ with a position dependent $\operatorname{coin} C(\boldsymbol{x}) \in U(2 d)$ and $d \geq 2$. However, for conceptual and notational simplicity, we first concentrate on the case of $d=2$. The case of $d \geq 3$ is dealt with in the subsequent sections. Let $\mathcal{H}=\ell^{2}\left(\mathbb{Z}^{2} ; \mathbb{C}^{4}\right)$ be the Hilbert space of states. As usual, the evolution operator $U=S C$ is defined as the product of a shift $S$ and a coin $C$. To define the shift operator, we introduce a set

$$
D=\left\{(\boldsymbol{p}, \boldsymbol{q})=\left(p_{1}, p_{2}, q_{1}, q_{2}\right) \in \mathbb{R}^{2} \times \mathbb{C}^{2}: p_{j}^{2}+\left|q_{j}\right|^{2}=1(j=1,2)\right\}
$$


and use $\left\{\boldsymbol{e}_{j}\right\}_{j=1}^{2}$ to denote the standard basis of $\mathbb{Z}^{2}$. We define operators $S_{j}(j=1,2)$ on $\ell^{2}\left(\mathbb{Z}^{2} ; \mathbb{C}^{2}\right)$ as

$$
\left(S_{j} \Psi\right)(\boldsymbol{x})=\left(\begin{array}{c}
p_{j} \psi_{1}(\boldsymbol{x})+q_{j} \psi_{2}\left(\boldsymbol{x}+\boldsymbol{e}_{j}\right) \\
q_{j}^{*} \psi_{1}\left(\boldsymbol{x}-\boldsymbol{e}_{j}\right)-p_{j} \psi_{2}(\boldsymbol{x})
\end{array}\right), \quad \boldsymbol{x} \in \mathbb{Z}^{2}
$$

for all $\Psi={ }^{t}\left(\psi_{1}, \psi_{2}\right) \in \ell^{2}\left(\mathbb{Z}^{2} ; \mathbb{C}^{2}\right)$. The shift $S$ on $\mathcal{H}$ is defined as a diagonal operator $S=S_{1} \oplus S_{2}$ on $\mathcal{H} \simeq \oplus^{2} \ell^{2}\left(\mathbb{Z}^{2} ; \mathbb{C}^{2}\right)$. The condition $(\boldsymbol{p}, \boldsymbol{q}) \in D$ ensures that $S_{j}$ is selfadjoint and unitary on $\ell^{2}\left(\mathbb{Z}^{2} ; \mathbb{C}^{2}\right)$ and so is $S$ on $\mathcal{H}$. The coin operator is a multiplication by unitary and self-adjoint square matrices $C(\boldsymbol{x}) \in U(4)$. In general, a unitary and selfadjoint operator is an involution; hence, it can only have eigenvalues \pm 1 as its spectrum. We impose the following on the coin operator $C$.

- (Simplicity) $\operatorname{dim} \operatorname{ker}(C(\boldsymbol{x})-1)=1, \quad \boldsymbol{x} \in \mathbb{Z}^{2}$.

- (One defect) $C(\boldsymbol{x})=\left\{\begin{array}{ll}C_{1}, & \boldsymbol{x} \in \mathbb{Z}^{2} \backslash\{\mathbf{0}\} \\ C_{0}, & \boldsymbol{x}=\mathbf{0}\end{array}\right.$ with some $C_{0}, C_{1} \in U(4)$.

We here comment on the aforementioned conditions. The simplicity condition means that $C(\boldsymbol{x})$ is a Grover-type coin. Indeed, by $\operatorname{dim} \operatorname{ker}(C(\boldsymbol{x})-1)=1$, we can take a unique normalized eigenvector $\chi(\boldsymbol{x}) \in \operatorname{ker}(C(\boldsymbol{x})-1)$ up to a constant factor. As seen in Lemma 3.1, we can write $C=2 d^{*} d-1$ with a coisometry $d: \mathcal{H} \rightarrow \mathcal{K}:=\ell^{2}\left(\mathbb{Z}^{2}\right)$ defined as

$$
(d \Psi)(\boldsymbol{x})=\langle\chi(\boldsymbol{x}), \Psi(\boldsymbol{x})\rangle_{\mathbb{C}^{2}}, \quad \boldsymbol{x} \in \mathbb{Z}^{2} \quad \text { for all } \Psi \in \mathcal{H} .
$$

The one defect condition means that $\chi(\boldsymbol{x})$ can be written as

$$
\chi(\boldsymbol{x})= \begin{cases}\Phi={ }^{t}\left(\Phi_{1}, \Phi_{2}\right) \text { with } \Phi_{j} \in \mathbb{C}^{2}(j=1,2), & \boldsymbol{x} \in \mathbb{Z}^{2} \backslash\{\mathbf{0}\}, \\ \Omega={ }^{t}\left(\Omega_{1}, \Omega_{2}\right) \text { with } \Omega_{j} \in \mathbb{C}^{2}(j=1,2), & \boldsymbol{x}=\mathbf{0},\end{cases}
$$

where $\Phi \in \operatorname{ker}\left(C_{1}-1\right)$ and $\Omega \in \operatorname{ker}\left(C_{0}-1\right)$ are normalized vectors. In Grover's search algorithm on a graph $G=(V, E)$, the coin operator $C(\boldsymbol{x})$ differs only at a vertex $\boldsymbol{x}=$ $\boldsymbol{x}_{0}$, which is a unique solution to the search problem. This is a one-defect condition. Moreover, finding the marked vertex $\boldsymbol{x}_{0}$ with non-zero probability is closely related to localization of the corresponding QW. Motivated by Grover's search algorithm, we study localization of the one defect model on $\mathbb{Z}^{d}$.

Results Let $\Psi_{0} \in \mathcal{H}$ be the initial state of a quantum walker, and let $\Psi_{t}=U^{t} \Psi_{0}$ $(t=1,2, \ldots)$ be the state of the walker at time $t$. The position $X_{t}$ of the walker at time $t$ follows $P\left(X_{t}=\boldsymbol{x}\right)=\left\|\Psi_{t}(\boldsymbol{x})\right\|_{\mathbb{C}^{2}}^{2}\left(\boldsymbol{x} \in \mathbb{Z}^{2}\right)$. As shown in [34], if the initial state $\Psi_{0}$ has a overlap with an eigenvector of $U$, then localization occurs, i.e.,

$$
\limsup _{t \rightarrow \infty} P\left(X_{t}=\boldsymbol{x}\right)>0 \text { with some } \boldsymbol{x} \in \mathbb{Z}^{2} .
$$

Thus the problem is reduced to proving the existence of eigenvalues for $U$. 
We are now in a position to state our result. Let $\sigma_{1}=\left(\begin{array}{ll}0 & 1 \\ 1 & 0\end{array}\right), \sigma_{+}=\left(\begin{array}{ll}0 & 1 \\ 0 & 0\end{array}\right)$, and $\sigma_{3}=\left(\begin{array}{cc}1 & 0 \\ 0 & -1\end{array}\right)$. We set $a_{\Omega}(\boldsymbol{p})=\sum_{j=1}^{2} p_{j}\left\langle\Omega_{j}, \sigma_{3} \Omega_{j}\right\rangle_{\mathbb{C}^{2}}, a_{\Phi}(\boldsymbol{p})=\sum_{j=1}^{2} p_{j}\left\langle\Phi_{j}, \sigma_{3} \Phi_{j}\right\rangle_{\mathbb{C}^{2}}$, $\lambda(\boldsymbol{q})=2 \sum_{j=1}^{2}\left|q_{j}\left\langle\Phi_{j}, \sigma_{+} \Phi_{j}\right\rangle_{\mathbb{C}^{2}}\right|$, and $D_{j}=\left\{(\boldsymbol{p}, \boldsymbol{q}) \in D: p_{j} q_{j} \neq 0\right\}(j=1,2)$. Let $\mathbb{T}_{-}=$ $\left[-1,-\lambda(\boldsymbol{q})+a_{\Phi}(\boldsymbol{p})\right), \mathbb{T}_{+}=\left(\lambda(\boldsymbol{q})+a_{\Phi}(\boldsymbol{p}), 1\right]$, and $g_{ \pm}(\lambda)=e^{ \pm i \arccos \lambda}$. We use $\cdot$ to denote the scalar product.

Theorem 1.1. Let $U=S C$ as above. Suppose that the following conditions hold.

(1) $\Phi_{j} \cdot\left(\sigma_{1} \Omega_{j}\right)=0$ for all $j \in\{1,2\}$ and $\left\langle\Phi_{l}, \sigma_{+} \Omega_{l}\right\rangle_{\mathbb{C}^{2}} \neq 0$ with some $l \in\{1,2\}$;

(2) $a_{\Omega}\left(\boldsymbol{p}_{0}\right) \neq a_{\Phi}\left(\boldsymbol{p}_{0}\right)$ with some $\boldsymbol{p}_{0} \in\{-1,1\} \times\{-1,1\}$.

If $(\boldsymbol{p}, \boldsymbol{q}) \in D_{l}$ and $\left\|(\boldsymbol{p}, \boldsymbol{q})-\left(\boldsymbol{p}_{0}, \mathbf{0}\right)\right\|_{\mathbb{R}^{2} \times \mathbb{C}^{2}}$ is sufficiently small, $U$ has two eigenvalues in $\left\{g_{-}(\lambda), g_{+}(\lambda) \mid \lambda \in \mathbb{T}_{-}\right\}$or $\left\{g_{-}(\lambda), g_{+}(\lambda) \mid \lambda \in \mathbb{T}_{+}\right\}$.

This is a special case of Theorem 3.7. See Figure 1 for the location of the eigenvalues and the continuous spectrum. The criteria for $U_{\mathrm{I}}$ to have eigenvalues in $\left\{g_{-}(\lambda), g_{+}(\lambda) \mid\right.$ $\left.\lambda \in \mathbb{T}_{-}\right\}$and $\left\{g_{-}(\lambda), g_{+}(\lambda) \mid \lambda \in \mathbb{T}_{+}\right\}$are obtained in Theorem 3.5 .

Methods and related work Localization of the one defect model of traditional onedimensional QWs was solved by Cantero et al [7], who used the CGMV method, which is not applicable for multidimensional cases. In the present work, we use the SMT. Several studies on the birth eigenspace $\mathcal{D}_{\mathrm{B}}$ have been reported. As shown by Higuchi et al [16], multi-dimensional models are likely to have eigenvalues \pm 1 due to the existence of cycles, which makes $\mathcal{D}_{\mathrm{B}}$ non trivial. For the one-dimensional split-step QW, the birth eigenspace is characterized elsewhere [12]. However, the eigenspace contained in the in-

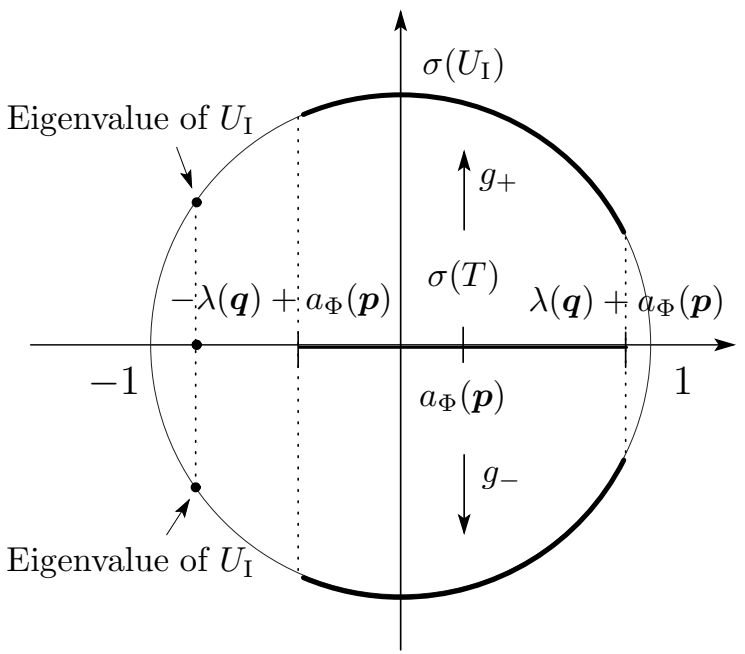

Figure 1: Location of the spectrum $\sigma\left(U_{\mathrm{I}}\right)$ for $a_{\Omega}\left(\boldsymbol{p}_{0}\right)<a_{\Phi}\left(\boldsymbol{p}_{0}\right) . \quad g_{ \pm}(\lambda)=e^{ \pm i \arccos \lambda}$ $\operatorname{map} \sigma(T)=\left[-\lambda(\boldsymbol{q})+a_{\Phi}(\boldsymbol{p}), \lambda(\boldsymbol{q})+a_{\Phi}(\boldsymbol{p})\right]$ onto $\sigma\left(U_{\mathrm{I}}\right) \subset S^{1}$. The difference $\sigma(U) \backslash$ $\sigma\left(U_{\mathrm{I}}\right)$ is at most $\sigma\left(U_{\mathrm{B}}\right) \subset\{-1,+1\}$. See Theorem 3.7 for more details. herited subspace $\mathcal{D}_{\mathrm{I}}$ is study only for the Grover walk case, in which the discriminant $T$ is unitarily equivalent to the transition probability matrix $P_{G}$ of the symmetric random walk on $G$. In our case, $T$ becomes a 
discrete Schrödinger operator with variable coefficients:

$$
T=a(\boldsymbol{p}, \cdot)+\sum_{j=1}^{2}\left\{q_{j}\left\langle\chi_{j}, L_{j} \sigma_{+} \chi_{j}\right\rangle+\left(q_{j}\left\langle\chi_{j}, L_{j} \sigma_{+} \chi_{j}\right\rangle\right)^{*}\right\},
$$

where $\chi(\boldsymbol{x})={ }^{t}\left(\chi_{1}(\boldsymbol{x}), \chi_{2}(\boldsymbol{x})\right), a(\boldsymbol{p}, \boldsymbol{x})=\sum_{j=1}^{2} p_{j}\left\langle\chi_{j}(\boldsymbol{x}), \sigma_{3} \chi_{j}(\boldsymbol{x})\right\rangle$, and $L_{j}$ is the shift by $\boldsymbol{e}_{j}$ on $\mathcal{K}$. To analyze the above operator $T$, we employ the Feshbach map $[9]$ :

$$
F(\lambda)=\Pi^{\perp}(T-\lambda) \Pi^{\perp}-\Pi^{\perp} T \Pi(\Pi(T-\lambda) \Pi)_{\operatorname{ran} \Pi}^{-1} \Pi T \Pi^{\perp}, \quad \lambda \in \mathbb{C} \backslash\left\{a_{\Omega}(\boldsymbol{p})\right\},
$$

where $\Pi$ is the projection onto $\{\psi \in \mathcal{K} \mid \psi(\boldsymbol{x})=0$ except for $\boldsymbol{x}=0\}$ and $\lambda$ is a spectral parameter. The isospectral property of this map implies that $\lambda$ is an eigenvalue of $T$ if $\operatorname{ker} F(\lambda)$ is non-trivial (Proposition 4.2). The Feshbach map was used in a study of nuclear reactions [9] and was used for constructing a renormalization map [6]. To our best knowledge, this is the first application of the Feshbach map to analyze the spectrum of an evolution operator for a QW. The one defect condition yields the following formula:

$$
F(\lambda)=\Pi^{\perp}\left(T_{0}-\lambda-\frac{1}{a_{\Omega}(\boldsymbol{p})-\lambda}\left|\varphi_{\boldsymbol{q}}\right\rangle\left\langle\varphi_{\boldsymbol{q}}\right|\right) \Pi^{\perp},
$$

where $\varphi_{\boldsymbol{q}} \in \mathcal{K}$. This is a one rank perturbation of a constant coefficient discrete Laplacian $T_{0}$. The spectral analysis of an operator $(1 / \sqrt{d}) \sum_{j=1}^{d}\left(L_{j}+L_{j}^{*}\right)+v\left|\delta_{\mathbf{0}}\right\rangle\left\langle\delta_{\mathbf{0}}\right|$ (with $v$ a coupling constant and $\delta_{\mathbf{0}}$ the delta function at the origin) similar to the right-hand side in (1.1) is treated elsewhere [19]. Because the nonlinearity of the spectral parameter $\lambda$, the analysis of the kernel of $F(\lambda)$ becomes more involved. This task is reduced to finding zeros of a function

$$
\mathfrak{f}(\lambda)=\lambda-a_{\Omega}(\boldsymbol{p})+\left\langle\varphi_{\boldsymbol{q}},\left(T_{0}-\lambda\right)^{-1} \varphi_{\boldsymbol{q}}\right\rangle_{\mathcal{K}}, \quad \lambda \in[-1,1] \backslash \sigma\left(T_{0}\right) \neq \emptyset .
$$

The rest of this paper is constructed as follows. In Sec. 2, we review the SMT, which plays an important role in this work. The precise definitions of our evolution $U$ and the discriminant $T$ are given in Sec. 3. We thereafter give the essential spectrum of $T$, which is mapped onto the essential spectrum of $U_{\mathrm{I}}$ by the SMT. We also give a criterion for $T$ to have an eigenvalue in terms of the Feshbach map $F(\lambda)$ (Theorem [3.4). We then present the main results. Theorem 3.5 gives criteria for $U$ to have eigenvalues and Theorem 3.7 shows the existence of eigenvalues for $U$. We prove Theorem 3.7 using Theorem 3.5. Sec. 4 is devoted to the precise definition of the Feshbach map $F(\lambda)$ and its properties. Sec. 5 is devoted to the analysis of $\mathfrak{f}(\lambda)$ and the proof of Theorem 3.5 .

\section{Preliminaries}

In this section, we briefly review the spectral mapping theorem (SMT). Readers can consult [18, 34] for more details. We use $\sigma(A), \sigma_{\mathrm{p}}(A), \sigma_{\mathrm{ac}}(A), \sigma_{\mathrm{sc}}(A)$ to denote the spectrum, the set of all eigenvalues, the absolutely continuous spectrum, and the singular 
continuous spectrum of an operator $A$, respectively. Let $\mathcal{H}$ and $\mathcal{K}$ be Hilbert spaces and $d: \mathcal{H} \rightarrow \mathcal{K}$ be a coisometry, i.e.,

$$
d d^{*}=I_{\mathcal{K}},
$$

where $d^{*}: \mathcal{K} \rightarrow \mathcal{H}$ is the adjoint of $d$ and $I_{\mathcal{K}}$ is the identity on $\mathcal{K}$. Then, $d$ is an isometry and $d^{*} d$ is a othogonal projection on $\mathcal{H}$, because $d^{*} d$ is idempotent and self-adjoint, i.e., $\left(d^{*} d\right)^{2}=d^{*} d$ and $\left(d^{*} d\right)^{*}=d^{*} d$. The operator

$$
C:=2 d^{*} d-1
$$

is a self-adjoint unitary operator, because $C^{2}=1$. Let $S$ be a self-adjoint unitary operator on $\mathcal{H}$ and set $U=S C$. The discriminant operator $T$ of $U$ is defined as

$$
T=d S d^{*}
$$

which is a bounded self-adjoint operator on $\mathcal{K}$ and $\|T\| \leq 1$. Hence, $\sigma(T)$ is a closed set contained in the interval $[-1,1]$. Let $\mathcal{D}_{ \pm}=\operatorname{ker} d \cap \operatorname{ker}(S \pm 1) \subset \mathcal{H}$. The subspaces

$$
\mathcal{D}_{\mathrm{B}}:=\mathcal{D}_{+} \oplus \mathcal{D}_{-} \quad \text { and } \quad \mathcal{D}_{\mathrm{I}}:=\mathcal{D}_{\mathrm{B}}^{\perp}
$$

are called the birth eigenspace of $U$ and inherited subspace of $U$, respectively. The restriction $U_{\mathrm{I}}:=\left.U\right|_{\mathcal{D}_{\mathrm{I}}}$ to the inherited subspace is unitarily equivalent to

$$
\exp (+i \arccos T) \oplus \exp (-i \arccos T) \quad \text { on } \operatorname{ran}\left(d^{*} d\right)
$$

See [34] for the precise meaning of the above decomposition. On the other hand, the restriction $U_{\mathrm{B}}:=\left.U\right|_{\mathcal{D}_{\mathrm{B}}}$ to the birth eigenspace coincides with $I_{\mathcal{D}_{+}} \oplus\left(-I_{\mathcal{D}_{-}}\right)$. The SMT from $T$ to $U$ is given as follows.

Theorem 2.1 (Spectral mapping theorem [18, 34]). Let $U=S C$ be as above. Then, $U$ is decomposed into $U=U_{\mathrm{I}} \oplus U_{\mathrm{B}}$ on $\mathcal{H}=\mathcal{D}_{\mathrm{I}} \oplus \mathcal{D}_{\mathrm{B}}$ and the following hold:

(1) $\sigma_{\sharp}(U)=\sigma_{\sharp}\left(U_{\mathrm{I}}\right)$ for $\sharp=\mathrm{ac}$, sc and $\sigma_{\mathrm{p}}(U)=\sigma_{\mathrm{p}}\left(U_{\mathrm{I}}\right) \cup \sigma_{\mathrm{p}}\left(U_{\mathrm{B}}\right)$;

(2) $\sigma_{\sharp}\left(U_{\mathrm{I}}\right)=\exp \left(+i \arccos \sigma_{\sharp}(T)\right) \cup \exp \left(-i \arccos \sigma_{\sharp}(T)\right)$ for $\sharp=\mathrm{p}, \mathrm{ac}, \mathrm{sc}$;

(3) $\sigma_{\sharp}\left(U_{\mathrm{B}}\right)=\emptyset$ for $\sharp=$ ac, sc and $\sigma_{\mathrm{p}}\left(U_{\mathrm{B}}\right)= \begin{cases}\{1,-1\} & \text { if } \mathcal{D}_{+} \neq \emptyset \text { and } \mathcal{D}_{-} \neq \emptyset \text {, } \\ \{ \pm 1\} & \text { if } \mathcal{D}_{ \pm} \neq \emptyset \text { and } \mathcal{D}_{\mp}=\emptyset \text {, } \\ \emptyset & \text { otherwise. }\end{cases}$

Theorem 2.1 is widely applicable for the evolutions of quantum walks. Here we give two examples. The first one is the Szegedy walk. See [16] for the twisted Szegedy walk.

Example 2.1 ([16, 18]). Let $D$ be the set of arcs of a symmetric digraph $G=(V, D)$ (possibly not bipartite) and $\mathcal{H}=\ell^{2}(D)$ be the Hilbert space of square summable functions $\psi: D \rightarrow \mathbb{C}$. Define a unitary operator $U_{G}$ on $\ell^{2}(D)$ as the product

$$
U_{G}=S_{\mathrm{f}} C_{\chi}
$$


of a shift $S_{\mathrm{f}}$ and coin $C_{\chi}$. The shift $S_{\mathrm{f}}$ is defined as $\left(S_{\mathrm{f}} \psi\right)(e)=\psi(\bar{e})$ for $e \in D$, where $\bar{e}$ stands for the inverse arc of e. The coin $C_{\chi}$ is defined as

$$
C_{\chi}=\bigoplus_{x \in V}(2|\chi(x)\rangle\langle\chi(x)|-1)
$$

where we have used an identification $\ell^{2}(D) \simeq \bigoplus_{x \in V} \mathcal{H}_{x}$ with $\mathcal{H}_{x}=\overline{\operatorname{Span}}\{\psi: \psi(e)=$ $0, o(e) \neq x\}$ and $\chi(x)=\sum_{e \in D ; o(e)=x} \sqrt{p_{t(e), x}} \delta_{e} \in \ell^{2}(D)$ is a normalized vector. Here $p_{u, v}$ is the transition probability of a (classical) random walk from $v$ to $u(u, v \in V)$. The $Q W$ with this evolution $U_{G}$ is now referred to as the Szegedy walk on $G$, which is called the Grover walk on $G$ in particular if $p_{u, v}=1 / \operatorname{deg} x$. In the case of the Szegedy evolution operator $U_{G}$, Theorem 2.1 is applicable for any symmetric digraph $G=(V, D)$, because $S_{\mathrm{f}}$ is self-adjoint and unitary and $C_{\chi}=2 d_{\chi}^{*} d_{\chi}-1$ with a coisometry $d_{\chi}: \ell^{2}(D) \rightarrow \ell^{2}(V)$ defined as

$$
\left(d_{\chi} \psi\right)(x):=\langle\chi(x), \psi\rangle, \quad x \in V
$$

Moreover, the discriminant operator $T_{G}$ of $U_{G}$ is unitary equivalent to the transition probability matrix $P_{G}=\left(p_{u, v}\right)$ and the birth eigenspace can be characterized by the structure of $G$.

The next example is a one-dimensional QW but not the Szegedy walk on $\mathbb{Z}$. This is a unified model including a split-step QW introduced by Kitagawa et al [23] and traditional one-dimensional QWs [2, 13, 28] as special cases. In the subsequent sections, we consider a multidimensional extension of this model.

Example 2.2 (Split-step QWs). The evolution of a split-step $Q W$ is a unitary operator on $\ell^{2}\left(\mathbb{Z} ; \mathbb{C}^{2}\right)$ defined as $U=S_{1} C$, where

$$
\left(S_{1} \psi\right)(x)=\left(\begin{array}{c}
p \psi_{1}(x)+q \psi_{2}(x+1) \\
q^{*} \psi_{1}(x-1)-p_{2} \psi(x)
\end{array}\right), \quad x \in \mathbb{Z}
$$

We suppose that $(p, q) \in \mathbb{R} \times \mathbb{C}$ satisfy $p^{2}+|q|^{2}=1$, which ensure $S_{1}$ is unitary and self-adjoint. $C$ is a multiplication operator by unitary matrices $C(x) \in U(2)$. When $p=0$ and $q=1$, it becomes a $Q W$ on $\mathbb{Z}$ with a flip-flop shift [5], which is unitarily equivalent to traditional $Q W s$ (see [31] for more information). The evolutions with $p=0$ and $p \neq 0$ are not unitarily equivalent and these walks have weak limit measures different from usual one [11]. If $C(x)$ is self-adjoint unitary and $\operatorname{dim} \operatorname{ker}(C(x)-1)=1$ for all $x \in \mathbb{Z}$, then $C$ is written as $2 d_{\chi}^{*} d_{\chi}-1$ with a coisometry $d_{\chi}: \ell^{2}\left(\mathbb{Z} ; \mathbb{C}^{2}\right) \rightarrow \ell^{2}(\mathbb{Z})$ defined as

$$
\left(d_{\chi} \Psi\right)(x)=\langle\chi(x), \Psi(x)\rangle_{\mathbb{C}^{2}}, \quad x \in \mathbb{Z}
$$

where $\chi(x) \in \operatorname{ker}(C(x)-1)$. Thus the SMT is applicable for this model. In [12], the birth eigenspace of this model is characterized. 


\section{Multi-dimensional models and main results}

\subsection{Definition of models}

From now on, we consider a $\mathrm{QW}$ on $\mathbb{Z}^{d}$, which is a generalization of the split-step QW defined in Example 2.2. Let $n \in \mathbb{N}$ and use $\ell^{2}\left(\mathbb{Z}^{d} ; \mathbb{C}^{n}\right)$ to denote the Hilbert space of the square-summable functions $\Psi: \mathbb{Z}^{d} \rightarrow \mathbb{C}^{n}$. If $n=1$, we $\operatorname{simply} \operatorname{denote} \ell^{2}\left(\mathbb{Z}^{d} ; \mathbb{C}\right)$ by $\ell^{2}\left(\mathbb{Z}^{d}\right)$. Hereafter, we set $\mathcal{H}=\ell^{2}\left(\mathbb{Z}^{d} ; \mathbb{C}^{2 d}\right)$ and $\mathcal{K}=\ell^{2}\left(\mathbb{Z}^{d}\right)$. We fist define an evolution operator $U$ on $\mathcal{H}$ as a product $U=S C$ of a shift operator $S$ and coin operator $C$, then introduce a coisometry $d: \mathcal{H} \rightarrow \mathcal{K}$, and give an explicit formula of the discriminant operator $T=d S d^{*}$ on $\mathcal{K}$.

Shift operators Let

$$
D=\left\{(\boldsymbol{p}, \boldsymbol{q})=\left(p_{1}, \ldots, p_{d}, q_{1}, \ldots, q_{d}\right) \in \mathbb{R}^{d} \times \mathbb{C}^{d}: p_{j}^{2}+\left|q_{j}\right|^{2}=1(j=1, \ldots, d)\right\}
$$

and use $\left\{\boldsymbol{e}_{j}\right\}_{j=1}^{d}$ to denote the standard basis of $\mathbb{Z}^{d}$. Henceforth $(\boldsymbol{p}, \boldsymbol{q}) \in D$ is assumed unless otherwise specified. To define a shift operator $S$ on $\mathcal{H}$, we introduce an operator $S_{j}$ on $\ell^{2}\left(\mathbb{Z}^{d} ; \mathbb{C}^{2}\right)(j=1, \ldots, d)$ as follows.

$$
\left(S_{j} \psi\right)(\boldsymbol{x})=\left(\begin{array}{c}
p_{j} \psi_{1}(\boldsymbol{x})+q_{j} \psi_{2}\left(\boldsymbol{x}+\boldsymbol{e}_{j}\right) \\
q_{j}^{*} \psi_{1}\left(\boldsymbol{x}-\boldsymbol{e}_{j}\right)-p_{j} \psi_{2}(\boldsymbol{x})
\end{array}\right) \quad \text { for all } \boldsymbol{x} \in \mathbb{Z}^{d} \text { and } \psi=\left(\begin{array}{l}
\psi_{1} \\
\psi_{2}
\end{array}\right) \in \ell^{2}\left(\mathbb{Z}^{d} ; \mathbb{C}^{2}\right)
$$

Using the identification $\mathcal{H} \simeq \oplus_{j=1}^{d} \ell^{2}\left(\mathbb{Z}^{d} ; \mathbb{C}^{2}\right)$, we define the shift $S$ on $\mathcal{H}$ as $S=S_{1} \oplus \ldots \oplus$ $S_{d}$, i.e.,

$$
(S \Psi)(\boldsymbol{x})=\left(\begin{array}{c}
\left(S_{1} \Psi_{1}\right)(\boldsymbol{x}) \\
\vdots \\
\left(S_{d} \Psi_{d}\right)(\boldsymbol{x})
\end{array}\right) \text { for all } \boldsymbol{x} \in \mathbb{Z}^{d} \text { and } \Psi=\left(\begin{array}{c}
\Psi_{1} \\
\vdots \\
\Psi_{d}
\end{array}\right) \in \mathcal{H}\left(\Psi_{j} \in \ell^{2}\left(\mathbb{Z}^{d} ; \mathbb{C}^{2}\right)\right)
$$

The condition $(\boldsymbol{p}, \boldsymbol{q}) \in D$ ensures $S_{j}$ is self-adjoint and unitary on $\ell^{2}\left(\mathbb{Z}^{d} ; \mathbb{C}^{2}\right)$, and so is $S$ on $\mathcal{H}$. Let $\{C(\boldsymbol{x})\}_{\boldsymbol{x} \in \mathbb{Z}^{d}} \subset U(2 d)$ be a family of unitary and self-adjoint square matrices of order $2 d$.

Coin operators We define a coin operator $C$ on $\mathcal{H}$ as a multiplication operator by $C(x)$, i.e.,

$$
(C \Psi)(\boldsymbol{x})=C(\boldsymbol{x}) \Psi(\boldsymbol{x}) \quad \text { for all } \boldsymbol{x} \in \mathbb{Z}^{d} \text { and } \Psi \in \mathcal{H} .
$$

By definition, $C$ is unitary on $\mathcal{H}$. Throughout this paper, the following two conditions are imposed on $C$ unless otherwise specified.

- (Simplicity) Each $C(\boldsymbol{x})$ has 1 as a simple eigenvalue, i.e.,

$$
\operatorname{dim} \operatorname{ker}(C(\boldsymbol{x})-1)=1, \quad \boldsymbol{x} \in \mathbb{Z}^{d} .
$$


- (One defect) There exist matrices $C_{0}$ and $C_{1} \in U(2 d)$ such that

$$
C(\boldsymbol{x})= \begin{cases}C_{1}, & \boldsymbol{x} \in \mathbb{Z}^{d} \backslash\{\mathbf{0}\} \\ C_{0}, & \boldsymbol{x}=\mathbf{0}\end{cases}
$$

Because dim $\operatorname{ker}(C(\boldsymbol{x})-1)=1$, we can take a unique normalized vector (up to a constant factor):

$$
\chi(\boldsymbol{x})=\left(\begin{array}{c}
\chi_{1}(\boldsymbol{x}) \\
\vdots \\
\chi_{d}(\boldsymbol{x})
\end{array}\right) \in \operatorname{ker}(C(\boldsymbol{x})-1), \quad \chi_{j}(\boldsymbol{x})=\left(\begin{array}{c}
\chi_{j, 1}(\boldsymbol{x}) \\
\chi_{j, 2}(\boldsymbol{x})
\end{array}\right) \in \mathbb{C}^{2}(j=1, \cdots, d) .
$$

The spectral decomposition of $C(\boldsymbol{x})$ implies $C(\boldsymbol{x})=2|\chi(\boldsymbol{x})\rangle\langle\chi(\boldsymbol{x})|-1$. By the one defect condition of $C, \chi(\boldsymbol{x})$ is written as follows.

$$
\chi(\boldsymbol{x})=\left\{\begin{array}{c}
\Phi=\left(\begin{array}{c}
\Phi_{1} \\
\vdots \\
\Phi_{d}
\end{array}\right) \text { with } \Phi_{j}=\left(\begin{array}{c}
\phi_{j, 1} \\
\phi_{j, 2}
\end{array}\right) \in \mathbb{C}^{2}(j=1, \cdots, d), \quad \boldsymbol{x} \in \mathbb{Z}^{d} \backslash\{\boldsymbol{0}\}, \\
\Omega=\left(\begin{array}{c}
\Omega_{1} \\
\vdots \\
\Omega_{d}
\end{array}\right) \text { with } \Omega_{j}=\left(\begin{array}{c}
\omega_{j, 1} \\
\omega_{j, 2}
\end{array}\right) \in \mathbb{C}^{2}(j=1, \cdots, d), \quad \boldsymbol{x}=\mathbf{0} .
\end{array}\right.
$$

Evolutions and their discriminants Let $S$ and $C$ be as above and define an evolution operator $U$ on $\mathcal{H}$ as

$$
U=S C
$$

$S$ and $C$ are unitary, and so is $U$. We define a coisometry $d: \mathcal{H} \rightarrow \mathcal{K}$ as

$$
(d \Psi)(\boldsymbol{x})=\langle\chi(\boldsymbol{x}), \Psi(\boldsymbol{x})\rangle_{\mathbb{C}^{2 d}} \quad \text { for all } \boldsymbol{x} \in \mathbb{Z}^{d} \text { and } \Psi \in \mathcal{H}
$$

Lemma 3.1. (1) The adjoint $d^{*}: \mathcal{K} \rightarrow \mathcal{H}$ of $d$ is a multiplication operator by $\chi(\boldsymbol{x})$, i.e.,

$$
\left(d^{*} f\right)(\boldsymbol{x})=\chi(\boldsymbol{x}) f(\boldsymbol{x}) \quad \text { for all } \boldsymbol{x} \in \mathbb{Z}^{d} \text { and } f \in \mathcal{K}
$$

(2) $d^{*} d=\bigoplus_{\boldsymbol{x} \in \mathbb{Z}^{d}}|\chi(\boldsymbol{x})\rangle\langle\chi(\boldsymbol{x})|$ and $d d^{*}=I_{\mathcal{K}}$.

(3) $C=2 d^{*} d-1$.

Proof. (1) For all $f \in \mathcal{K}$, since $\sum_{\boldsymbol{x} \in \mathbb{Z}^{d}}\|\chi(\boldsymbol{x}) f(\boldsymbol{x})\|_{\mathbb{C}^{2 d}}^{2}=\sum_{\boldsymbol{x} \in \mathbb{Z}^{d}}|f(\boldsymbol{x})|^{2}=\|f\|_{\mathcal{K}}^{2}<\infty$, then the multiplication operator $\chi: \mathcal{K} \ni f \mapsto \chi f \in \mathcal{H}$ is bounded. For all $\Psi \in \mathcal{H}$ and $f \in \mathcal{K}$, $\langle f, d \Psi\rangle_{\mathcal{K}}=\sum_{\boldsymbol{x} \in \mathbb{Z}^{d}} f(\boldsymbol{x})^{*}\langle\chi(\boldsymbol{x}), \Psi(\boldsymbol{x})\rangle_{\mathbb{C}^{2 d}}=\langle\chi f, \Psi\rangle_{\mathcal{H}}$. Thus we have $d^{*} f=\chi f$. 
(2) For all $\Psi \in \mathcal{H}$ and $\boldsymbol{x} \in \mathbb{Z}^{d},\left(d^{*} d \Psi\right)(\boldsymbol{x})=\chi(\boldsymbol{x})(d \Psi)(\boldsymbol{x})=\langle\chi(\boldsymbol{x}), \Psi(\boldsymbol{x})\rangle \chi(\boldsymbol{x})$ holds. Then we have $d^{*} d=\bigoplus_{\boldsymbol{x} \in \mathbb{Z}^{d}}|\chi(\boldsymbol{x})\rangle\langle\chi(\boldsymbol{x})|$. On the other hand, for all $f \in \mathcal{K}$ and $\boldsymbol{x} \in \mathbb{Z}^{d}$, $\left(d d^{*} f\right)(\boldsymbol{x})=\left\langle\chi(\boldsymbol{x}),\left(d^{*} f\right)(\boldsymbol{x})\right\rangle_{\mathbb{C}^{2 d}}=\langle\chi(\boldsymbol{x}), f(\boldsymbol{x}) \chi(\boldsymbol{x})\rangle_{\mathbb{C}^{2 d}}=f(\boldsymbol{x})$. Then $d d^{*}=I_{\mathcal{K}}$ holds.

(3) Obviously, the result follows from $d^{*} d=\bigoplus_{\boldsymbol{x} \in \mathbb{Z}^{d}}|\chi(\boldsymbol{x})\rangle\langle\chi(\boldsymbol{x})|$.

Lemma 3.1 implies that Theorem 2.1 is applicable for the above evolution $U$. In what follows, we give an explicit form of the discriminant operator $T$ of $U$, defined as

$$
T=d S d^{*}
$$

Let $L_{j}$ be a shift on $\mathcal{K}$ by $\boldsymbol{e}_{j}(j \in\{1, \cdots, d\})$, i.e.,

$$
\left(L_{j} f\right)(\boldsymbol{x})=f\left(\boldsymbol{x}+\boldsymbol{e}_{j}\right), \quad \text { for all } \boldsymbol{x} \in \mathbb{Z}^{d} \text { and } f \in \mathcal{K},
$$

by which $S_{j}$ can be expressed as a matrix form

$$
S_{j}=\left(\begin{array}{cc}
p_{j} I_{\mathcal{K}} & q_{j} L_{j} \\
q_{j}^{*} L_{j}^{*} & -p_{j} I_{\mathcal{K}}
\end{array}\right)
$$

We use the following notations:

$$
\begin{gathered}
a_{\Omega}(\boldsymbol{p})=\sum_{j=1}^{d} p_{j}\left\langle\Omega_{j}, \sigma_{3} \Omega_{j}\right\rangle_{\mathbb{C}^{2}}, \quad a_{\Phi}(\boldsymbol{p})=\sum_{j=1}^{d} p_{j}\left\langle\Phi_{j}, \sigma_{3} \Phi_{j}\right\rangle_{\mathbb{C}^{2}}, \\
\text { and } \quad a(\boldsymbol{p}, \boldsymbol{x})=\sum_{j=1}^{d} p_{j}\left\langle\chi_{j}(\boldsymbol{x}), \sigma_{3} \chi_{j}(\boldsymbol{x})\right\rangle_{\mathbb{C}^{2}},
\end{gathered}
$$

where $\sigma_{3}=\left(\begin{array}{cc}1 & 0 \\ 0 & -1\end{array}\right)$. Observe that

$$
a(\boldsymbol{p}, \boldsymbol{x})=a_{\Omega}(\boldsymbol{p}) \mathbb{1}_{\{\mathbf{0}\}}(\boldsymbol{x})+a_{\Phi}(\boldsymbol{p}) \mathbb{1}_{\mathbb{Z}^{d} \backslash\{\mathbf{0}\}}(\boldsymbol{x}),
$$

where $\mathbb{1}_{A}$ is the characteristic function of a set $A$. As seen in Section 2 , the discriminant operator $T=d S d^{*}$ of $U$ is a bounded self-adjoint on $\mathcal{K}$ and $\|T\| \leq 1$.

Lemma 3.2. $T$ is expressed as

$$
T=a(\boldsymbol{p}, \cdot)+\sum_{j=1}^{d}\left\{q_{j} \chi_{j, 1}^{*} L_{j} \chi_{j, 2}+\left(q_{j} \chi_{j, 1}^{*} L_{j} \chi_{j, 2}\right)^{*}\right\}
$$

where $\chi_{j, 1}, \chi_{j, 2}$ and $a(\boldsymbol{p}, \cdot)$ denote multiplication operators.

Remark 3.1. In Section 1, we abbreviate the expression (3.3) as

$$
T=a(\boldsymbol{p}, \cdot)+\sum_{j=1}^{2}\left\{q_{j}\left\langle\chi_{j}, L_{j} \sigma_{+} \chi_{j}\right\rangle+\left(q_{j}\left\langle\chi_{j}, L_{j} \sigma_{+} \chi_{j}\right\rangle\right)^{*}\right\} \text { with } \sigma_{+}=\left(\begin{array}{ll}
0 & 1 \\
0 & 0
\end{array}\right) \text {. }
$$


Proof. For all $f \in \mathcal{K}, j \in\{1, \cdots, d\}$, we set $\left(d^{*} f\right)_{j}=\chi_{j} f \in \ell^{2}\left(\mathbb{Z}^{d} ; \mathbb{C}^{2}\right)$. Since $d^{*} f=$ $\left(\begin{array}{c}\left(d^{*} f\right)_{1} \\ \vdots \\ \left(d^{*} f\right)_{d}\end{array}\right)$, we have $S d^{*} f=\left(\begin{array}{c}S_{1}\left(d^{*} f\right)_{1} \\ \vdots \\ S_{d}\left(d^{*} f\right)_{d}\end{array}\right)$. By definition of $T$, the following holds for all $f \in \mathcal{K}$ and $\boldsymbol{x} \in \mathbb{Z}^{d}$ :

$$
\begin{aligned}
(T f)(\boldsymbol{x}) & =\left\langle\chi(\boldsymbol{x}),\left(S d^{*} f\right)(\boldsymbol{x})\right\rangle_{\mathbb{C}^{2 d}}=\sum_{j=1}^{d}\left\langle\chi_{j}(\boldsymbol{x}),\left(S_{j}\left(d^{*} f\right)_{j}\right)(\boldsymbol{x})\right\rangle_{\mathbb{C}^{2}} \\
& =\sum_{j=1}^{d}\left\langle\left(\begin{array}{l}
\chi_{j, 1} \\
\chi_{j, 2}
\end{array}\right)(\boldsymbol{x}),\left(\left(\begin{array}{cc}
p_{j} & q_{j} L_{j} \\
q_{j}^{*} L_{j}^{*} & -p_{j}
\end{array}\right)\left(\begin{array}{l}
f \chi_{j, 1} \\
f \chi_{j, 2}
\end{array}\right)\right)(\boldsymbol{x})\right\rangle_{\mathbb{C}^{2}} \\
& =\left(\left(a(\boldsymbol{p}, \cdot)+\sum_{j=1}^{d}\left\{q_{j} \chi_{j, 1}^{*} L_{j} \chi_{j, 2}+\left(q_{j} \chi_{j, 1}^{*} L_{j} \chi_{j, 2}\right)^{*}\right\}\right) f\right)(\boldsymbol{x}) .
\end{aligned}
$$

We close this subsection by characterizing the essential spectrum of the discriminant $T$. To this ends, we introduce a self-adjoint operator $T_{0}$ and constant $\lambda(\boldsymbol{q})$ by

$$
T_{0}=a_{\Phi}(\boldsymbol{p})+\sum_{j=1}^{d}\left(\alpha_{j} L_{j}+\alpha_{j}^{*} L_{j}^{*}\right) \quad \text { and } \quad \lambda(\boldsymbol{q})=2 \sum_{j=1}^{d}\left|\alpha_{j}\right|,
$$

where $\alpha_{j}=q_{j} \phi_{j, 1}^{*} \phi_{j, 2}(j=1, \cdots, d)$. In Sec. 1 , we set $\lambda(\boldsymbol{q})=2 \sum_{j=1}^{2}\left|q_{j}\left\langle\Phi_{j}, \sigma_{+} \Phi_{j}\right\rangle_{\mathbb{C}^{2}}\right|$, because $\alpha_{j}=q_{j}\left\langle\Phi_{j}, \sigma_{+} \Phi_{j}\right\rangle_{\mathbb{C}^{2}}$.

Lemma 3.3. It follows that

$$
\sigma_{\mathrm{ess}}(T)=\sigma_{\mathrm{ess}}\left(T_{0}\right)=\sigma\left(T_{0}\right)=\left[-\lambda(\boldsymbol{q})+a_{\Phi}(\boldsymbol{p}), a_{\Phi}(\boldsymbol{p})+\lambda(\boldsymbol{q})\right] .
$$

Moreover, the following conditions are equivalent:

(1) $\sigma\left(T_{0}\right)=[-1,1]$;

(2) $\lambda(\boldsymbol{q})=1$;

(3) $p_{j}=0$ and $\left|\phi_{j, 1}\right|=\left|\phi_{j, 2}\right|$ for all $j \in\{1, \cdots, d\}$.

Remark 3.2. Let $g_{ \pm}(\lambda)=e^{ \pm i \arccos \lambda}$. The spectral mapping theorem (Theorem 2.1) concludes that

$$
\sigma_{\text {ess }}(U)=\left\{g_{-}(\lambda) \mid \lambda \in \sigma\left(T_{0}\right)\right\} \cup\left\{g_{+}(\lambda) \mid \lambda \in \sigma\left(T_{0}\right)\right\} .
$$

See Figure 1. 
Proof. Let $W=T-T_{0}$. Then $T=T_{0}+W$ and

$$
\begin{aligned}
W & =a(\boldsymbol{p}, \boldsymbol{x})-a_{\Phi}(\boldsymbol{p})+\sum_{j=1}^{d} q_{j}\left(\chi_{j, 1}(\boldsymbol{x})^{*} \chi_{j, 2}\left(\boldsymbol{x}+\boldsymbol{e}_{j}\right)-\phi_{j, 1}^{*} \phi_{j, 2}\right) L_{j} \\
& +\sum_{j=1}^{d} q_{j}^{*}\left(\chi_{j, 2}(\boldsymbol{x})^{*} \chi_{j, 1}\left(\boldsymbol{x}-\boldsymbol{e}_{j}\right)-\phi_{j, 2}^{*} \phi_{j, 1}\right) L_{j}^{*} .
\end{aligned}
$$

Because, by (3.1) and (3.2), $(W f)(\boldsymbol{x})=0$ for all $\boldsymbol{x} \neq \pm \boldsymbol{e}_{j}, \boldsymbol{0}$ and $f \in \mathcal{K}$,

$$
W=\beta_{0} \mathbb{1}_{\{\mathbf{0}\}}+\sum_{j=1}^{d}\left\{\beta_{j}^{+} \mathbb{1}_{\left\{\boldsymbol{e}_{j}\right\}}+\beta_{j}^{-} \mathbb{1}_{\left\{-\boldsymbol{e}_{j}\right\}}\right\}
$$

with some constants $\beta_{0}$ and $\beta_{j}^{ \pm}(j=1, \ldots, d)$. Because $W$ is compact, $\sigma_{\text {ess }}(T)=\sigma_{\text {ess }}\left(T_{0}\right)$.

Let $\mathcal{F}: \mathcal{K} \rightarrow L^{2}\left([0,2 \pi]^{d}, d \boldsymbol{k} /(2 \pi)^{d}\right)$ be the Fourier transformation defined as the unitary extension of

$$
(\mathcal{F} f)(\boldsymbol{k})=\hat{f}(\boldsymbol{k})=\sum_{\boldsymbol{x} \in \mathbb{Z}^{d}} e^{-i \boldsymbol{k} \cdot \boldsymbol{x}} f(\boldsymbol{x}) \quad \text { for all } f \in \mathcal{K} \text { with finite support. }
$$

Because $\mathcal{F} L_{j} \mathcal{F}^{*}$ and $\mathcal{F} L_{j}^{*} \mathcal{F}^{*}$ are multiplication operators by $e^{i k_{j}}$ and $e^{-i k_{j}}$, the Fourier transform $\mathcal{F}_{0} \mathcal{F}^{*}$ of $T_{0}$ is also a multiplication operator by

$$
\hat{T}_{0}(\boldsymbol{k})=a_{\Phi}(\boldsymbol{p})+2 \sum_{j=1}^{d}\left|\alpha_{j}\right| \cos \left(k_{j}+\theta_{j}\right),
$$

where each $\theta_{j} \in[0,2 \pi)$ is an argument of $\alpha_{j}$, i.e., $\alpha_{j}=\left|\alpha_{j}\right| e^{i \theta_{j}}$ (if $\alpha_{j}=0$, we define $\left.\theta_{j}=0\right)$. Because $\hat{T}_{0}\left([0,2 \pi]^{d}\right)=\left[-\lambda_{\boldsymbol{q}}+a_{\Phi}(\boldsymbol{p}), a_{\Phi}(\boldsymbol{p})+\lambda_{\boldsymbol{q}}\right]$, we have (3.4).

(3.4) implies that $a_{\Phi}(\boldsymbol{p})=0$ if $\lambda(\boldsymbol{q})=1$ and hence that $\sigma\left(T_{0}\right)=[-1,1]$ if and only if $\lambda(\boldsymbol{q})=1$. On the other hand, $\left|q_{j}\right| \leq 1$ and the inequality of arithmetic and geometric means yield the inequality

$$
\lambda(\boldsymbol{q}) \leq 2 \sum_{j=1}^{d}\left|\phi_{j, 1} \phi_{j, 2}\right| \leq \sum_{j=1}^{d}\left(\left|\phi_{j, 1}\right|^{2}+\left|\phi_{j, 2}\right|^{2}\right)=1
$$

with equality if and only if $\left|q_{j}\right|=1$ and $\left|\phi_{j, 1}\right|=\left|\phi_{j, 2}\right|$ for all $j \in\{1, \cdots, d\}$. This completes the proof.

\subsection{Main results}

In what follows, we prove the existence of discrete eigenvalues of $U_{\mathrm{I}}$. To this end, we impose the following on the coin operator, which corresponds to (1) in Theorem 1.1 for the case of $d=2$. Let $\sigma_{1}=\left(\begin{array}{ll}0 & 1 \\ 1 & 0\end{array}\right)$. We use - to denote the scalar product, i.e., $\Psi \cdot \Phi=\psi_{1} \phi_{1}+\psi_{2} \phi_{2}$ for $\Psi={ }^{t}\left(\psi_{1}, \psi_{2}\right), \Phi={ }^{t}\left(\phi_{1}, \phi_{2}\right) \in \mathbb{C}^{2}$. 
Assumption 1. (a) $\Phi_{j} \cdot\left(\sigma_{1} \Omega_{j}\right)=0$ for all $j \in\{1, \cdots, d\}$;

(b) $\left\langle\Phi_{l}, \sigma_{+} \Omega_{l}\right\rangle_{\mathbb{C}^{2}} \neq 0$ with some $l \in\{1, \cdots, d\}$.

Let $l$ be as in Assumption 1 and set

$$
D_{l}=\left\{(\boldsymbol{p}, \boldsymbol{q}) \in D: p_{l} q_{l} \neq 0\right\} .
$$

Lemma 3.3 shows that if $(\boldsymbol{p}, \boldsymbol{q}) \in D_{l}$, then $\sigma_{\text {ess }}(T)=\sigma\left(T_{0}\right) \subsetneq[-1,1]$. Hence, there can exist discrete eigenvalues of $T$ in $[-1,1] \backslash \sigma\left(T_{0}\right) \neq \emptyset$. In order to find the discrete eigenvalue, we introduce a function $\mathfrak{f}:[-1,1] \backslash \sigma\left(T_{0}\right) \rightarrow \mathbb{R}$ as follows. Let

$$
\varphi_{\boldsymbol{q}}=\sum_{j=1}^{d}\left(q_{j} \omega_{j, 2} \phi_{j, 1}^{*} \mathbb{1}_{\left\{-\boldsymbol{e}_{j}\right\}}+q_{j}^{*} \omega_{j, 1} \phi_{j, 2}^{*} \mathbb{1}_{\left\{\boldsymbol{e}_{j}\right\}}\right) \in \mathcal{K} .
$$

For $\lambda \in[-1,1] \backslash \sigma\left(T_{0}\right) \neq \emptyset$, we define

$$
\mathfrak{f}(\lambda)=\lambda-a_{\Omega}(\boldsymbol{p})+\left\langle\varphi_{\boldsymbol{q}}, \psi_{\lambda}\right\rangle_{\mathcal{K}}
$$

where

$$
\psi_{\lambda}=\left(T_{0}-\lambda\right)^{-1} \varphi_{\boldsymbol{q}} \in \mathcal{K} .
$$

Let $\sigma_{-}=\sigma_{+}^{*}$. Because $\varphi_{\boldsymbol{q}}$ is written as

$$
\varphi_{\boldsymbol{q}}=\sum_{j=1}^{d}\left(q_{j}\left\langle\Phi_{j}, \sigma_{+} \Omega_{j}\right\rangle \mathbb{1}_{\left\{-\boldsymbol{e}_{j}\right\}}+q_{j}^{*}\left\langle\Phi_{j}, \sigma_{-} \Omega_{j}\right\rangle \mathbb{1}_{\left\{\boldsymbol{e}_{j}\right\}}\right)
$$

$(\boldsymbol{p}, \boldsymbol{q}) \in D_{l}$ ensures that $\varphi_{\boldsymbol{q}} \not \equiv 0$ and $\psi_{\lambda} \not \equiv 0$.

The next theorem plays an important role to show the eigenvalue of $T$.

Theorem 3.4. Suppose that Assumption 1 holds and $(\boldsymbol{p}, \boldsymbol{q}) \in D_{l}$. If $\mathfrak{f}$ has a zero $\lambda_{\star} \in$ $[-1,1] \backslash\left(\sigma\left(T_{0}\right) \cup\left\{a_{\Omega}(\boldsymbol{p})\right\}\right)$, then $\lambda_{\star}$ is a discrete eigenvalue of $T$.

Remark 3.3. By Lemma 5.2 (3), $a_{\Omega}(\boldsymbol{p})$ can not be a zero of $\mathfrak{f}$ even if $a_{\Omega}(\boldsymbol{p}) \in[-1,1] \backslash$ $\sigma\left(T_{0}\right)$. Hence, $\mathfrak{f}$ has a zero $\lambda_{\star} \in[-1,1] \backslash\left(\sigma\left(T_{0}\right) \cup\left\{a_{\Omega}(\boldsymbol{p})\right\}\right)$ (if it exists) and Theorem 3.4 concludes that $\lambda_{\star} \in \sigma_{\mathrm{p}}(U)$. The SMT and Theorem 3.4 imply that $g_{ \pm}\left(\lambda_{\star}\right) \in \sigma_{\mathrm{p}}(U)$ are discrete eigenvalues of $U$. See Figure 1 .

The proof of Theorem 3.4 is based on the Feshbach projection method [9, 6]. This reduces the spectral analysis of $T$ to that of the Feshbach map $F(T, P, \lambda)$, which is an operator defined by $T$, a projection $P$ suitably chosen, and a spectral parameter $\lambda$. Let $\Pi=\left|\mathbb{1}_{\{\mathbf{0}\}}\right\rangle\left\langle\mathbb{1}_{\{\mathbf{0}\}}\right|$ be the projection onto the subspace $\left\{\alpha \mathbb{1}_{\{\mathbf{0}\}} \mid \alpha \in \mathbb{C}\right\} \subset \mathcal{K}$ and $\Pi^{\perp}=$ $I_{\mathcal{K}}-\Pi$. Here we chose $P=\Pi^{\perp}$ as the projection defining the Feshbach map and set $F(\lambda)=F\left(T, \Pi^{\perp}, \lambda\right)$. See Sec. 4 for the precise definition of $F(\lambda)$ and propositions used in the following proof. 
Proof of Theorem 3.4. By Proposition 4.3, $F(\lambda)$ is written as

$$
F(\lambda)=\Pi^{\perp}\left(T_{0}-\lambda-\frac{1}{a_{\Omega}(\boldsymbol{p})-\lambda}\left|\varphi_{\boldsymbol{q}}\right\rangle\left\langle\varphi_{\boldsymbol{q}}\right|\right) \Pi^{\perp}, \quad \lambda \in \mathbb{C} \backslash\left\{a_{\Omega}(\boldsymbol{p})\right\} .
$$

Let $\lambda_{\star} \in[-1,1] \backslash\left(\sigma\left(T_{0}\right) \cup\left\{a_{\Omega}(\boldsymbol{p})\right\}\right)$ be a zero of $\mathfrak{f}$, i.e., $\mathfrak{f}\left(\lambda_{\star}\right)=0$, and let $\psi_{\lambda_{\star}}$ be defined in (3.6) with $\lambda=\lambda_{\star}$. Because by Proposition 4.4, $\psi_{\lambda_{\star}} \in \operatorname{ran} \Pi^{\perp} \backslash\{0\}$,

$$
\begin{aligned}
F\left(\lambda_{\star}\right) \psi_{\lambda_{\star}} & =\Pi^{\perp}\left(T_{0}-\lambda_{\star}-\frac{1}{a_{\Omega}(\boldsymbol{p})-\lambda_{\star}}\left|\varphi_{\boldsymbol{q}}\right\rangle\left\langle\varphi_{\boldsymbol{q}}\right|\right) \psi_{\lambda_{\star}} \\
& =\left(1-\frac{\left\langle\varphi_{\boldsymbol{q}}, \psi_{\lambda_{\star}}\right\rangle}{a_{\Omega}(\boldsymbol{p})-\lambda}\right) \varphi_{\boldsymbol{q}}=-\frac{\mathfrak{f}\left(\lambda_{\star}\right)}{a_{\Omega}(\boldsymbol{p})-\lambda_{\star}} \varphi_{\boldsymbol{q}}=0 .
\end{aligned}
$$

This completes the proof, because by Proposition $4.2, \lambda_{\star} \in \sigma_{\mathrm{p}}(T)$ is equivalent that ker $F\left(\lambda_{\star}\right)$ is non trivial, which is confirmed by Proposition 4.4 again.

The following is a criterion for $\mathfrak{f}$ to have a zero.

Theorem 3.5. Suppose that Assumption 1 holds and $(\boldsymbol{p}, \boldsymbol{q}) \in D_{l}$.

(1) $\mathfrak{f}$ has a zero $\lambda_{\star} \in \mathbb{T}_{-}:=\left[-1,-\lambda(\boldsymbol{q})+a_{\Phi}(\boldsymbol{p})\right)$ if

$$
\lambda(\boldsymbol{q})\left(\lambda(\boldsymbol{q})+a_{\Omega}(\boldsymbol{p})-a_{\Phi}(\boldsymbol{p})\right)<\left\|\varphi_{\boldsymbol{q}}\right\|^{2} \leq\left(1+a_{\Omega}(\boldsymbol{p})\right) \frac{\left(1+a_{\Phi}(\boldsymbol{p})\right)^{2}-\lambda(\boldsymbol{q})^{2}}{1+a_{\Phi}(\boldsymbol{p})}
$$

(2) $\mathfrak{f}$ has a zero $\lambda_{\star} \in \mathbb{T}_{+}=\left(\lambda(\boldsymbol{q})+a_{\Phi}(\boldsymbol{p})\right.$, 1] if

$$
\lambda(\boldsymbol{q})\left(\lambda(\boldsymbol{q})-a_{\Omega}(\boldsymbol{p})+a_{\Phi}(\boldsymbol{p})\right)<\left\|\varphi_{\boldsymbol{q}}\right\|^{2} \leq\left(1-a_{\Omega}(\boldsymbol{p})\right) \frac{\left(1-a_{\Phi}(\boldsymbol{p})\right)^{2}-\lambda(\boldsymbol{q})^{2}}{1-a_{\Phi}(\boldsymbol{p})} .
$$

Thanks to Lemma 3.6 below, the right-hand sides of (3.8) and (3.9) make sense. The proof of Theorem 3.5 will be stated in the last section.

Lemma 3.6. Suppose that Assumption 1 holds and $(\boldsymbol{p}, \boldsymbol{q}) \in D_{l}$. Then,

$$
a_{\Phi}(\boldsymbol{p}) \neq \pm 1 \quad \text { and } \quad a_{\Omega}(\boldsymbol{p}) \neq \pm 1 .
$$

Proof. Suppose $a_{\Phi}(\boldsymbol{p})=-1$. By the definition of $a_{\Phi}(\boldsymbol{p})$ and $\|\Phi\|^{2}=1$,

$$
-1=\sum_{j \notin A} p_{j}\left(\left|\phi_{j, 1}\right|^{2}-\left|\phi_{j, 2}\right|^{2}\right) \quad \text { and } \quad 1=\sum_{j \notin A}\left(\left|\phi_{j, 1}\right|^{2}+\left|\phi_{j, 2}\right|^{2}\right)
$$

where $A=\left\{j \in\{1, \cdots, d\} \mid \phi_{j, 1}=\phi_{j, 2}=0\right\}$. Summing the above two equations, we get $0=\sum_{j \notin A}\left\{\left(1+p_{j}\right)\left|\phi_{j, 1}\right|^{2}+\left(1-p_{j}\right)\left|\phi_{j, 2}\right|^{2}\right\}$, which, combined with $1 \pm p_{j} \geq 0$, implies that for $j \notin A$,

$$
\left(1-p_{j}\right)\left|\phi_{j, 2}\right|^{2}=0 .
$$

By Assumption 1 and $(\boldsymbol{p}, \boldsymbol{q}) \in D_{l}, \phi_{l, 2} \neq 0, p_{l} \neq 1$, and hence $l \notin A$. This contradicts (3.10). Therefore $a_{\Phi}(\boldsymbol{p}) \neq-1$. The remainder can be shown similarly. 
To state our main result, we introduce the following assumption.

Assumption 2. $a_{\Omega}\left(\boldsymbol{p}_{0}\right) \neq a_{\Phi}\left(\boldsymbol{p}_{0}\right)$ holds with some $\boldsymbol{p}_{0} \in\{-1,1\}^{d}$.

Remark 3.4. If $d=1$, then Assumptions 1 1 [2 are not compatible. See [12] for $d=1$.

Theorem 3.7 (Existence of eigenvalues). Let $d \geq 2$ and suppose that Assumptions 1 , 2 holds. Then, there exists $\delta>0$ such that if $(\boldsymbol{p}, \boldsymbol{q}) \in D_{l}$ satisfies $\left\|(\boldsymbol{p}, \boldsymbol{q})-\left(\boldsymbol{p}_{0}, \mathbf{0}\right)\right\|_{\mathbb{R}^{d} \times \mathbb{C}^{d}}<\delta$, then there exist eigenvalues of $U$. Moreover, the following hold.

(1) If, in addition, $a_{\Omega}\left(\boldsymbol{p}_{0}\right)<a_{\Phi}\left(\boldsymbol{p}_{0}\right)$, then $g_{-}\left(\lambda_{\star}\right)$ and $g_{+}\left(\lambda_{\star}\right)$ are eigenvalues of $U_{\mathrm{I}}$ with some $\lambda_{\star} \in \mathbb{T}_{-}$;

(2) If, in addition, $a_{\Omega}\left(\boldsymbol{p}_{0}\right)>a_{\Phi}\left(\boldsymbol{p}_{0}\right)$, then $g_{-}\left(\lambda_{\star}\right)$ and $g_{+}\left(\lambda_{\star}\right)$ are eigenvalues of $U_{\mathrm{I}}$ with some $\lambda_{\star} \in \mathbb{T}_{+}$.

Proof. Observe that $\varphi_{\boldsymbol{q}} \neq 0$ and $\lambda(\boldsymbol{q})>0$ whenever $(\boldsymbol{p}, \boldsymbol{q}) \in D_{l}$. By continuity, we have

$$
\lim _{\boldsymbol{q} \rightarrow \mathbf{0}}\|\varphi(\boldsymbol{q})\|=\lim _{\boldsymbol{q} \rightarrow \mathbf{0}} \lambda(\boldsymbol{q})=0, \lim _{\boldsymbol{p} \rightarrow \boldsymbol{p}_{0}} a_{\Omega}(\boldsymbol{p})=a_{\Omega}\left(\boldsymbol{p}_{0}\right), \text { and } \lim _{\boldsymbol{p} \rightarrow \boldsymbol{p}_{0}} a_{\Phi}(\boldsymbol{p})=a_{\Phi}\left(\boldsymbol{p}_{0}\right) .
$$

Suppose that $a_{\Omega}\left(\boldsymbol{p}_{0}\right)<a_{\Phi}\left(\boldsymbol{p}_{0}\right)$. Then, there exists $\delta_{0}>0$ such that if $(\boldsymbol{p}, \boldsymbol{q}) \in D_{l}$ and $\left\|(\boldsymbol{p}, \boldsymbol{q})-\left(\boldsymbol{p}_{0}, \mathbf{0}\right)\right\|_{\mathbb{R}^{d} \times \mathbb{C}^{d}}<\delta_{0}$, then $\lambda(\boldsymbol{q})\left(\lambda(\boldsymbol{q})+a_{\Omega}(\boldsymbol{p})-a_{\Phi}(\boldsymbol{p})\right)<0$. By Lemma 3.6 and (3.11),

$$
\lim _{(\boldsymbol{p}, \boldsymbol{q}) \rightarrow\left(\boldsymbol{p}_{0}, \mathbf{0}\right)}\left(1+a_{\Omega}(\boldsymbol{p})\right) \frac{\left(1+a_{\Phi}(\boldsymbol{p})\right)^{2}-\lambda(\boldsymbol{q})^{2}}{1+a_{\Phi}(\boldsymbol{p})}=\left(1+a_{\Omega}\left(\boldsymbol{p}_{0}\right)\right)\left(1+a_{\Phi}\left(\boldsymbol{p}_{0}\right)\right)>0 .
$$

Hence (3.8) holds if $(\boldsymbol{p}, \boldsymbol{q}) \in D_{l}$ satisfies $\left\|(\boldsymbol{p}, \boldsymbol{q})-\left(\boldsymbol{p}_{0}, \mathbf{0}\right)\right\|_{\mathbb{R}^{d} \times \mathbb{C}^{d}}<\delta$ with some $\delta>0$. Similarly, $a_{\Omega}\left(\boldsymbol{p}_{0}\right)>a_{\Phi}\left(\boldsymbol{p}_{0}\right)$ concludes that (3.9) holds. Applying Theorems 2.1 and 3.5 completes the proof.

Remark 3.5. Theorem 3.7 has demonstrated the existence of eigenvalues of $U_{\mathrm{I}}$ for sufficiently small $\boldsymbol{q}$. It would be interesting to study the existence of eigenvalues of $U_{\mathrm{I}}$ without such a condition.

Example 3.1. Let $d=2$ and set

$$
\Phi:=\frac{1}{\sqrt{2}}\left(\begin{array}{l}
1 \\
1 \\
0 \\
0
\end{array}\right), \quad \Omega:=\frac{1}{2}\left(\begin{array}{c}
1 \\
-1 \\
\sqrt{2} \\
0
\end{array}\right), \quad \boldsymbol{p}_{0}=(1,1) .
$$

Then, $\frac{1}{2}=a_{\Omega}\left(\boldsymbol{p}_{0}\right)>a_{\Phi}\left(\boldsymbol{p}_{0}\right)=0$ and all assumptions in Theorem 3.7 are satisfied with $l=1$. Hence $U$ has two eigenvalues if $(\boldsymbol{p}, \boldsymbol{q}) \in D_{1}$ and $\left\|(\boldsymbol{p}, \boldsymbol{q})-\left(\boldsymbol{p}_{0}, \mathbf{0}\right)\right\|$ is sufficiently small. More precisely, $g_{ \pm}\left(\lambda_{\star}\right) \in \sigma_{\mathrm{p}}\left(U_{\mathrm{I}}\right)$ with some $\lambda_{\star} \in \mathbb{T}_{+}$if $\boldsymbol{p}$ satisfies

$$
p_{2}<\frac{5}{2}-\frac{1}{2 p_{1}^{2}}, \quad 1<p_{1}^{2}+\frac{4}{9} p_{2}^{2} .
$$

This is because, in this case, (3.12) is equivalent to (3.9) in Theorem 3.5. 


\section{$4 \quad$ Feshbach map}

\subsection{Definition of the Feshbach map}

In this subsection, we define the Feshbach map of the discriminant operator $T$. Recall that $\Pi=\left|\mathbb{1}_{\{\mathbf{0}\}}\right\rangle\left\langle\mathbb{1}_{\{\mathbf{0}\}}\right|$ and $\Pi^{\perp}=I_{\mathcal{K}}-\Pi$. Let $\lambda \in \mathbb{C}$ and $(\Pi(T-\lambda) \Pi)_{\text {ran } \Pi \text { be a following }}$ operator on ranП:

$$
(\Pi(T-\lambda) \Pi)_{\operatorname{ran} \Pi}: \operatorname{ran} \Pi \ni f \mapsto(\Pi(T-\lambda) \Pi) f \in \operatorname{ran} \Pi .
$$

Lemma 4.1. The following conditions are equivalent:

(1) $\lambda \neq a_{\Omega}(\boldsymbol{p})$;

(2) There exists an inverse operator of $(\Pi(T-\lambda) \Pi)_{\mathrm{ran} \Pi \text {. }}$

In this case,

$$
(\Pi(T-\lambda) \Pi)_{\mathrm{ran} \Pi}^{-1}=\frac{1}{a_{\Omega}(\boldsymbol{p})-\lambda} I_{\mathrm{ran} \Pi,}
$$

where $I_{\text {ranח }}$ is an identity map on ranП.

Proof. Simple calculation show that $\left\langle\mathbb{1}_{\{\mathbf{0}\}}, \chi_{j, 1}^{*} L_{j} \chi_{j, 2} \mathbb{1}_{\{\mathbf{0}\}}\right\rangle=0$ for all $j \in\{1, \cdots, d\}$ and $\Pi(T-\lambda) \Pi=\left(a_{\Omega}(\boldsymbol{p})-\lambda\right) \Pi$ for all $\lambda \in \mathbb{C}$. Hence,

$$
(\Pi(T-\lambda) \Pi)_{\mathrm{ran} \Pi}=\left(a_{\Omega}(\boldsymbol{p})-\lambda\right) I_{\mathrm{ran} \Pi} \quad \text { for all } \lambda \in \mathbb{C} .
$$

Therefore, (1) and (2) are equivalent and (4.1) holds for $\lambda \neq a_{\Omega}(\boldsymbol{p})$.

Lemma 4.1 guarantees that the operator

$$
F(\lambda)=\Pi^{\perp}(T-\lambda) \Pi^{\perp}-\Pi^{\perp} T \Pi(\Pi(T-\lambda) \Pi)_{\mathrm{ran} \Pi}^{-1} \Pi T \Pi^{\perp}
$$

is well-defined whenever $\lambda \in \mathbb{C} \backslash\left\{a_{\Omega}(\boldsymbol{p})\right\}$. $F(\lambda)$ is called the Feshbach map of $T$. The following proposition reveals an isospectral property of the Feshbach map.

Proposition 4.2. Let $\lambda \in \mathbb{C} \backslash\left\{a_{\Omega}(\boldsymbol{p})\right\}$. Then, the following are equivalent:

(1) $\lambda \in \sigma_{\mathrm{p}}(T)$;

(2) $\operatorname{ker} F(\lambda)$ is non trivial.

In this case, $\operatorname{dim} \operatorname{ker}(T-\lambda)=\operatorname{dim} \operatorname{ker} F(\lambda)$.

Proof. See 6].

Proposition 4.3. Let $\lambda \in \mathbb{C} \backslash\left\{a_{\Omega}(\boldsymbol{p})\right\}$. Then, $F(\lambda)$ is written as

$$
F(\lambda)=\Pi^{\perp}\left(T_{0}-\lambda-\frac{1}{a_{\Omega}(\boldsymbol{p})-\lambda}\left|\varphi_{\boldsymbol{q}}\right\rangle\left\langle\varphi_{\boldsymbol{q}}\right|\right) \Pi^{\perp} .
$$


Proof. A simple calculation yields $\Pi T \Pi^{\perp}=\left|\mathbb{1}_{\{\mathbf{0}\}}\right\rangle\left\langle\varphi_{\boldsymbol{q}}\right|$. By definition,

$$
\begin{aligned}
F(\lambda) & =\Pi^{\perp}\left\{T-\lambda-\frac{1}{a_{\Omega}(\boldsymbol{p})-\lambda}\left(\Pi T \Pi^{\perp}\right)^{*}\left(\Pi T \Pi^{\perp}\right)\right\} \Pi^{\perp} \\
& =\Pi^{\perp}\left(T-\lambda-\frac{1}{a_{\Omega}(\boldsymbol{p})-\lambda}\left|\varphi_{\boldsymbol{q}}\right\rangle\left\langle\varphi_{\boldsymbol{q}}\right|\right) \Pi^{\perp} .
\end{aligned}
$$

It suffices to show $\Pi^{\perp} T \Pi^{\perp}=\Pi^{\perp} T_{0} \Pi^{\perp}$. By Lemma 3.2 ,

$$
\Pi^{\perp} T \Pi^{\perp}=\Pi^{\perp} a(\boldsymbol{p}, \cdot) \Pi^{\perp}+\sum_{j=1}^{d}\left\{q_{j} \Pi^{\perp} \chi_{j, 1}^{*} L_{j} \chi_{j, 2} \Pi^{\perp}+\left(q_{j} \Pi^{\perp} \chi_{j, 1}^{*} L_{j} \chi_{j, 2} \Pi^{\perp}\right)^{*}\right\}
$$

The first term of the right-hand side of (4.2) is calculated as

$$
\begin{aligned}
\Pi^{\perp} a(\boldsymbol{p}, \cdot) \Pi^{\perp} & =\sum_{j=1}^{d} p_{j} \Pi^{\perp}\left(\left|\chi_{j, 1}\right|^{2}-\left|\chi_{j, 2}\right|^{2}\right) \Pi^{\perp} \\
& =\sum_{j=1}^{d} p_{j} \sum_{\boldsymbol{x} \neq \mathbf{0}} \sum_{\boldsymbol{y} \neq \mathbf{0}}\left|\mathbb{1}_{\{\boldsymbol{x}\}}\right\rangle\left\langle\mathbb{1}_{\{\boldsymbol{x}\}},\left(\left|\chi_{j, 1}(\boldsymbol{x})\right|^{2}-\left|\chi_{j, 2}(\boldsymbol{x})\right|^{2}\right) \mathbb{1}_{\{\boldsymbol{y}\}}\right\rangle\left\langle\mathbb{1}_{\{\boldsymbol{y}\}}\right| \\
& =\sum_{j=1}^{d} p_{j} \sum_{\boldsymbol{x} \neq \mathbf{0}} \sum_{\boldsymbol{y} \neq \mathbf{0}}\left|\mathbb{1}_{\{\boldsymbol{x}\}}\right\rangle\left\langle\mathbb{1}_{\{\boldsymbol{x}\}},\left(\left|\phi_{j, 1}\right|^{2}-\left|\phi_{j, 2}\right|^{2}\right) \mathbb{1}_{\{\boldsymbol{y}\}}\right\rangle\left\langle\mathbb{1}_{\{\boldsymbol{y}\}}\right| \\
& =\Pi^{\perp} a_{\Phi}(\boldsymbol{p}) \Pi^{\perp} .
\end{aligned}
$$

On the other hand,

$$
\begin{aligned}
\Pi^{\perp} \chi_{j, 1}^{*} L_{j} \chi_{j, 2} \Pi^{\perp} & =\sum_{\boldsymbol{x} \neq \mathbf{0}} \sum_{\boldsymbol{y} \neq \mathbf{0}}\left|\mathbb{1}_{\{\boldsymbol{x}\}}\right\rangle\left\langle\mathbb{1}_{\{\boldsymbol{x}\}}, \chi_{j, 1}^{*} L_{j} \chi_{j, 2} \mathbb{1}_{\{\boldsymbol{y}\}}\right\rangle\left\langle\mathbb{1}_{\{\boldsymbol{y}\}}\right| \\
& =\sum_{\boldsymbol{x} \neq \mathbf{0}} \sum_{\boldsymbol{y} \neq \mathbf{0}}\left(\chi_{j, 1}^{*} L_{j} \chi_{j, 2} \mathbb{1}_{\{\boldsymbol{y}\}}\right)(\boldsymbol{x})\left|\mathbb{1}_{\{\boldsymbol{x}\}}\right\rangle\left\langle\mathbb{1}_{\{\boldsymbol{y}\}}\right| \\
& =\sum_{\boldsymbol{x} \neq \mathbf{0}} \sum_{\boldsymbol{y} \neq \mathbf{0}} \chi_{j, 1}^{*}(\boldsymbol{x}) \chi_{j, 2}\left(\boldsymbol{x}+\boldsymbol{e}_{j}\right) \mathbb{1}_{\{\boldsymbol{y}\}}\left(\boldsymbol{x}+\boldsymbol{e}_{j}\right)\left|\mathbb{1}_{\{\boldsymbol{x}\}}\right\rangle\left\langle\mathbb{1}_{\{\boldsymbol{y}\}}\right| \\
& =\sum_{\boldsymbol{x} \neq \mathbf{0}} \phi_{j, 1}^{*} \phi_{j, 2}\left|\mathbb{1}_{\{\boldsymbol{x}\}}\right\rangle\left\langle\mathbb{1}_{\left\{\boldsymbol{x}+\boldsymbol{e}_{j}\right\}}\right| \\
& =\Pi^{\perp} \phi_{j, 1}^{*} \phi_{j, 2} L_{j} \Pi^{\perp} .
\end{aligned}
$$

Hence, $\Pi^{\perp} T \Pi^{\perp}=\Pi^{\perp} T_{0} \Pi^{\perp}$. This completes the proof.

Remark 4.1. In the proof of Proposition 4.3, one defect condition plays an essential role. If the coin has two or more defect, then we can not conclude $\Pi^{\perp} \chi_{j, 1}^{*} L_{j} \chi_{j, 2} \Pi^{\perp}=$ $\Pi^{\perp} \phi_{j, 1}^{*} \phi_{j, 2} L_{j} \Pi^{\perp}$. 


\subsection{Non-triviality of the kernel of $F(\lambda)$}

The following proposition ensures the non-triviality of ker $F(\lambda)$ in the proof of Theorem 3.4. Recall that $\psi_{\lambda}=\left(T_{0}-\lambda\right)^{-1} \varphi_{\boldsymbol{q}} \in \mathcal{K}$.

Proposition 4.4. Suppose that Assumption 1 holds and $(\boldsymbol{p}, \boldsymbol{q}) \in D_{l}$. Then, $\psi_{\lambda} \in \operatorname{ran} \Pi^{\perp} \backslash$ $\{0\}$ for all $\lambda \in[-1,1] \backslash \sigma\left(T_{0}\right)$.

To prove Proposition 4.4, we need the following lemma.

Lemma 4.5. The following are equivalent:

(1) $\psi_{\lambda} \in \operatorname{ran} \Pi^{\perp}$;

(2) $\int_{[0,2 \pi]^{d}} \hat{\psi}_{\lambda}(\boldsymbol{k}) \frac{d \boldsymbol{k}}{(2 \pi)^{d}}=0$.

Proof. Since $\Pi^{\perp} \psi_{\lambda}=\psi_{\lambda}$ is equivalent that $\mathcal{F} \Pi^{\perp} \mathcal{F}^{*} \hat{\psi}_{\lambda}=\hat{\psi}_{\lambda}$, then (1) is equivalent the following:

$$
\hat{\psi}_{\lambda} \in \operatorname{ran} \mathcal{F} \Pi^{\perp} \mathcal{F}^{*} .
$$

Because by direct calculation, $\mathcal{F}_{\{\mathbf{0}\}}=\mathbb{1}_{[0,2 \pi]^{d}}$, the following holds:

$$
\begin{aligned}
\mathcal{F} \Pi^{\perp} \mathcal{F}^{*} \hat{\psi}_{\lambda} & =\mathcal{F}\left(I_{\mathcal{K}}-\Pi\right) \mathcal{F}^{*} \hat{\psi}_{\lambda} \\
& =\left(I_{\mathcal{F} \mathcal{K}}-\left|\mathbb{1}_{[0,2 \pi]^{d}}\right\rangle\left\langle\mathbb{1}_{[0,2 \pi] d}\right|\right) \hat{\psi}_{\lambda} \\
& =\hat{\psi}_{\lambda}-\left(\int_{[0,2 \pi]^{d}} \hat{\psi}_{\lambda}(\boldsymbol{k}) \frac{d \boldsymbol{k}}{(2 \pi)^{d}}\right) \mathbb{1}_{[0,2 \pi]^{d}} .
\end{aligned}
$$

By (4.3) and (4.4), (1) holds if and only if $\left(\int_{[0,2 \pi]^{d}} \hat{\psi}_{\lambda}(\boldsymbol{k}) \frac{d \boldsymbol{k}}{(2 \pi)^{d}}\right) \mathbb{1}_{[0,2 \pi]^{d}}=0$. This proves the lemma.

Proof of Proposition 4.4. Fix $\lambda \in[-1,1] \backslash \sigma\left(T_{0}\right)$. Because $\lambda \notin \sigma\left(T_{0}\right)$ and $\varphi_{\boldsymbol{q}} \neq 0$, we observe that $\psi_{\lambda} \neq 0$. By using (3.5) and changing variables, we have

$$
\begin{aligned}
& \int_{[0,2 \pi]^{d}} \hat{\psi}_{\lambda}(\boldsymbol{k}) \frac{d \boldsymbol{k}}{(2 \pi)^{d}}=\int_{[0,2 \pi]^{d}} \frac{\hat{\varphi}_{\boldsymbol{q}}(\boldsymbol{k})}{\hat{T}_{0}(\boldsymbol{k})-\lambda} \frac{d \boldsymbol{k}}{(2 \pi)^{d}} \\
& =\int_{[0,2 \pi]^{d}} \frac{\sum_{j=1}^{d}\left(\varphi_{\boldsymbol{q}}\left(\boldsymbol{e}_{j}\right) e^{-i k_{j}}+\varphi_{\boldsymbol{q}}\left(-\boldsymbol{e}_{j}\right) e^{i k_{j}}\right)}{a_{\Phi}(\boldsymbol{p})+2 \sum_{j=1}^{d}\left|\alpha_{j}\right| \cos \left(k_{j}+\theta_{j}\right)-\lambda} \frac{d \boldsymbol{k}}{(2 \pi)^{d}} \\
& =\int_{\prod_{j=1}^{d}\left[\theta_{j}, 2 \pi+\theta_{j}\right]} \frac{\sum_{j=1}^{d}\left(\varphi_{\boldsymbol{q}}\left(\boldsymbol{e}_{j}\right) e^{-i\left(t_{j}-\theta_{j}\right)}+\varphi_{\boldsymbol{q}}\left(-\boldsymbol{e}_{j}\right) e^{i\left(t_{j}-\theta_{j}\right)}\right)}{a_{\Phi}(\boldsymbol{p})+2 \sum_{j=1}^{d}\left|\alpha_{j}\right| \cos t_{j}-\lambda} \frac{d \boldsymbol{t}}{(2 \pi)^{d}} \\
& =\int_{[0,2 \pi]^{d}} \frac{\sum_{j=1}^{d}\left(\varphi_{\boldsymbol{q}}\left(\boldsymbol{e}_{j}\right) e^{i \theta_{j}}+\varphi_{\boldsymbol{q}}\left(-\boldsymbol{e}_{j}\right) e^{-i \theta_{j}}\right) \cos t_{j}}{a_{\Phi}(\boldsymbol{p})+2 \sum_{j=1}^{d}\left|\alpha_{j}\right| \cos t_{j}-\lambda} \frac{d \boldsymbol{t}}{(2 \pi)^{d}} .
\end{aligned}
$$


By (3.7), we observe that

$$
\varphi_{\boldsymbol{q}}\left(\boldsymbol{e}_{j}\right) e^{i \theta_{j}}+\varphi_{\boldsymbol{q}}\left(-\boldsymbol{e}_{j}\right) e^{-i \theta_{j}}=q_{j}^{*} e^{i \theta_{j}}\left\langle\Phi_{j}, \sigma_{-} \Omega_{j}\right\rangle,+q_{j} e^{-i \theta_{j}}\left\langle\Phi_{j}, \sigma_{+} \Omega_{j}\right\rangle .
$$

Let $B=\left\{j \mid q_{j} \neq 0,\left\langle\Phi_{j}, \sigma_{+} \Omega_{j}\right\rangle \neq 0\right\}$. Assumption $1(\mathrm{~b})$ and $(\boldsymbol{p}, \boldsymbol{q}) \in D_{l}$ imply $B \neq \emptyset$. If $j \notin B$, then the right-hand side (RHS) of (4.6) is zero, because by $\sigma_{1}=\sigma_{+}+\sigma_{-}$, Assumption 1 (a) implies that

$$
\left|\left\langle\Phi_{j}, \sigma_{+} \Omega_{j}\right\rangle\right|=\left|\Phi_{j} \cdot\left(\sigma_{+} \Omega_{j}\right)\right|=\left|\Phi_{j} \cdot\left(\sigma_{-} \Omega_{j}\right)\right|=\left|\left\langle\Phi_{j}, \sigma_{-} \Omega_{j}\right\rangle\right| .
$$

Let $j \in B$. By (4.7), we have $\phi_{j, 1} \neq 0, \phi_{j, 2} \neq 0$, and hence $\alpha_{j}=q_{j} \phi_{j, 1}^{*} \phi_{j, 2} \neq 0$. Using $e^{i \theta_{j}}=q_{j} \phi_{j, 1}^{*} \phi_{j, 2} /\left|\alpha_{j}\right|$, we observe that

$$
\begin{aligned}
\text { RHS of (4.6) } & =\left\langle\Phi_{j},\left(\begin{array}{cc}
0 & q_{j} e^{-i \theta_{j}} \\
q_{j}^{*} e^{i \theta_{j}} & 0
\end{array}\right) \Omega_{j}\right\rangle=\frac{\left|q_{j}\right|^{2}}{\left|\alpha_{j}\right|}\left\langle\left(\begin{array}{cc}
0 & \phi_{j, 1} \phi_{j, 2}^{*} \\
\phi_{j, 1}^{*} \phi_{j, 2} & 0
\end{array}\right) \Phi_{j}, \Omega_{j}\right\rangle \\
& =\frac{\left|q_{j}\right|^{2} \phi_{j, 1}^{*} \phi_{j, 2}^{*}}{\left|\alpha_{j}\right|} \Phi_{j} \cdot\left(\sigma_{1} \Omega_{j}\right)=0 .
\end{aligned}
$$

Therefore,

$$
\varphi_{\boldsymbol{q}}\left(\boldsymbol{e}_{j}\right) e^{i \theta_{j}}+\varphi_{\boldsymbol{q}}\left(-\boldsymbol{e}_{j}\right) e^{-i \theta_{j}}=0, \quad j=1, \ldots, d,
$$

which, in conjunction with (4.5), gives $\int_{[0,2 \pi]^{d}} \hat{\psi}_{\lambda}(\boldsymbol{k}) \frac{d \boldsymbol{k}}{(2 \pi)^{d}}=0$. Lemma 4.5 concludes the proof.

\section{Zeros of $\mathfrak{f}$}

In this section we prove Theorem 3.5. We henceforth suppose that Assumption 1 holds and fix $(\boldsymbol{p}, \boldsymbol{q}) \in D_{l}$. Recall that $\mathfrak{f}:[-1,1] \backslash \sigma\left(T_{0}\right) \rightarrow \mathbb{R}$ is defined as

$$
\mathfrak{f}(\lambda)=\lambda-a_{\Omega}(\boldsymbol{p})+\left\langle\varphi_{\boldsymbol{q}}, \psi_{\lambda}\right\rangle_{\mathcal{K}}
$$

and that we set $\mathbb{T}_{-}=\left[-1,-\lambda(\boldsymbol{q})+a_{\Phi}(\boldsymbol{p})\right)$ and $\mathbb{T}_{+}=\left(\lambda(\boldsymbol{q})+a_{\Phi}(\boldsymbol{p}), 1\right]$. We need the following lemmas.

Lemma 5.1. The function $\mathfrak{f}$ is continuously differentiable and monotonically increasing. Proof. The lemma is evident from

$$
\mathfrak{f}^{\prime}(\lambda)=1+\int_{[0,2 \pi]^{d}} \frac{\left|\hat{\varphi}_{\boldsymbol{q}}(\boldsymbol{k})\right|^{2}}{\left(\hat{T}_{0}(\boldsymbol{k})-\lambda\right)^{2}} \frac{d \boldsymbol{k}}{(2 \pi)^{d}}>0 .
$$

Lemma 5.2. The following hold: 
(1) If $\lambda \in \mathbb{T}_{-}$, then

$$
\lambda-a_{\Omega}(\boldsymbol{p})+\frac{\left\|\varphi_{\boldsymbol{q}}\right\|^{2}}{a_{\Phi}(\boldsymbol{p})-\lambda}<\mathfrak{f}(\lambda)<\lambda-a_{\Omega}(\boldsymbol{p})+\frac{a_{\Phi}(\boldsymbol{p})-\lambda}{\left(a_{\Phi}(\boldsymbol{p})-\lambda\right)^{2}-\lambda(\boldsymbol{q})^{2}}\left\|\varphi_{\boldsymbol{q}}\right\|^{2} .
$$

(2) If $\lambda \in \mathbb{T}_{+}$, then

$$
\lambda-a_{\Omega}(\boldsymbol{p})+\frac{\left\|\varphi_{\boldsymbol{q}}\right\|^{2}}{a_{\Phi}(\boldsymbol{p})-\lambda}>\mathfrak{f}(\lambda)>\lambda-a_{\Omega}(\boldsymbol{p})+\frac{a_{\Phi}(\boldsymbol{p})-\lambda}{\left(a_{\Phi}(\boldsymbol{p})-\lambda\right)^{2}-\lambda(\boldsymbol{q})^{2}}\left\|\varphi_{\boldsymbol{q}}\right\|^{2} .
$$

(3) If $a_{\Omega}(\boldsymbol{p}) \in[-1,1] \backslash \sigma\left(T_{0}\right)$, then $\mathfrak{f}\left(a_{\Omega}(\boldsymbol{p})\right) \neq 0$.

Proof. Let $\lambda \in \mathbb{T}_{-}$. Since $\varphi_{\boldsymbol{q}} \neq 0, \mathfrak{f}(\lambda)$ can be written as

$$
\mathfrak{f}(\lambda)=\lambda-a_{\Omega}(\boldsymbol{p})+\left\|\varphi_{\boldsymbol{q}}\right\|^{2} \int_{\sigma\left(T_{0}\right)} g_{\lambda}(x) d\left\langle\varphi_{\boldsymbol{q}}, E_{T_{0}}(x) \varphi_{\boldsymbol{q}}\right\rangle /\left\|\varphi_{\boldsymbol{q}}\right\|^{2},
$$

where $E_{T_{0}}(\cdot)$ is the spectral measure of $T_{0}$ and $g_{\lambda}(x)=\frac{1}{x-\lambda}$. Note that $\left\langle\varphi_{\boldsymbol{q}}, E_{T_{0}}(\cdot) \varphi_{\boldsymbol{q}}\right\rangle /\left\|\varphi_{\boldsymbol{q}}\right\|^{2}$ is a probability measure on $\sigma\left(T_{0}\right)=\left[a_{\Phi}(\boldsymbol{p})-\lambda_{\boldsymbol{q}}, a_{\Phi}(\boldsymbol{p})+\lambda_{\boldsymbol{q}}\right]$. By Jensen's inequality, we have

$$
\begin{aligned}
\int_{\sigma\left(T_{0}\right)} g_{\lambda}(x) d\left\langle\varphi_{\boldsymbol{q}}, E_{T_{0}}(x) \varphi_{\boldsymbol{q}}\right\rangle /\left\|\varphi_{\boldsymbol{q}}\right\|^{2} & >g_{\lambda}\left(\int_{\sigma\left(T_{0}\right)} x d\left\langle\varphi_{\boldsymbol{q}}, E_{T_{0}}(x) \varphi_{\boldsymbol{q}}\right\rangle /\left\|\varphi_{\boldsymbol{q}}\right\|^{2}\right) \\
& =g_{\lambda}\left(\left\langle\varphi_{\boldsymbol{q}}, T_{0} \varphi_{\boldsymbol{q}}\right\rangle /\left\|\varphi_{\boldsymbol{q}}\right\|^{2}\right) .
\end{aligned}
$$

Because $\left\langle\varphi_{\boldsymbol{q}}, T_{0} \varphi_{\boldsymbol{q}}\right\rangle /\left\|\varphi_{\boldsymbol{q}}\right\|^{2}=a_{\Phi}(\boldsymbol{p})$, we have,

$$
\mathfrak{f}(\lambda)>\lambda-a_{\Omega}(\boldsymbol{p})+\left\|\varphi_{\boldsymbol{q}}\right\|^{2} g_{\lambda}\left(a_{\Phi}(\boldsymbol{p})\right)=\lambda-a_{\Omega}(\boldsymbol{p})+\frac{\left\|\varphi_{\boldsymbol{q}}\right\|^{2}}{a_{\Phi}(\boldsymbol{p})-\lambda} .
$$

Let $u:\left[a_{\Phi}(\boldsymbol{p})-\lambda(\boldsymbol{q}), a_{\Phi}(\boldsymbol{p})+\lambda(\boldsymbol{q})\right] \rightarrow \mathbb{R}$ be a linear function such that $u\left(a_{\Phi}(\boldsymbol{p})-\lambda(\boldsymbol{q})\right)=$ $g_{\lambda}\left(a_{\Phi}(\boldsymbol{p})-\lambda(\boldsymbol{q})\right)$ and $u\left(a_{\Phi}(\boldsymbol{p})+\lambda(\boldsymbol{q})\right)=g_{\lambda}\left(a_{\Phi}(\boldsymbol{p})+\lambda(\boldsymbol{q})\right)$, i.e.,

$$
u(x)=\frac{-x+2 a_{\Phi}(\boldsymbol{p})-\lambda}{\left(a_{\Phi}(\boldsymbol{p})-\lambda\right)^{2}-\lambda(\boldsymbol{q})^{2}} .
$$

By the convexity of $g_{\lambda}$, we have

$$
\begin{aligned}
\int_{\sigma\left(T_{0}\right)} g_{\lambda}(x) d\left\langle\varphi_{\boldsymbol{q}}, E_{T_{0}}(x) \varphi_{\boldsymbol{q}}\right\rangle /\left\|\varphi_{\boldsymbol{q}}\right\|^{2} & <\int_{\sigma\left(T_{0}\right)} u(x) d\left\langle\varphi_{\boldsymbol{q}}, E_{T_{0}}(x) \varphi_{\boldsymbol{q}}\right\rangle /\left\|\varphi_{\boldsymbol{q}}\right\|^{2} \\
& =\frac{-\left\langle\varphi_{\boldsymbol{q}}, T_{0} \varphi_{\boldsymbol{q}}\right\rangle /\left\|\varphi_{\boldsymbol{q}}\right\|^{2}+2 a_{\Phi}(\boldsymbol{p})-\lambda}{\left(a_{\Phi}(\boldsymbol{p})-\lambda\right)^{2}-\lambda(\boldsymbol{q})^{2}} \\
& =\frac{a_{\Phi}(\boldsymbol{p})-\lambda}{\left(a_{\Phi}(\boldsymbol{p})-\lambda\right)^{2}-\lambda(\boldsymbol{q})^{2}} .
\end{aligned}
$$


Hence,

$$
\mathfrak{f}(\lambda)<\lambda-a_{\Omega}(\boldsymbol{p})+\frac{a_{\Phi}(\boldsymbol{p})-\lambda}{\left(a_{\Phi}(\boldsymbol{p})-\lambda\right)^{2}-\lambda_{\boldsymbol{q}}^{2}}\left\|\varphi_{\boldsymbol{q}}\right\|^{2} .
$$

(5.3) and (5.4) imply (5.1). Hence (1) is proved. The same proof works for (2).

We prove $(3)$. If $a_{\Omega}(\boldsymbol{p}) \in \mathbb{T}_{-}$, then, $a_{\Omega}(\boldsymbol{p})<a_{\Phi}(\boldsymbol{p})$. By (5.3), we have $\mathfrak{f}\left(a_{\Omega}(\boldsymbol{p})\right)>0$. Similarly, if $a_{\Omega}(\boldsymbol{p}) \in \mathbb{T}_{+}$, then $\mathfrak{f}\left(a_{\Omega}(\boldsymbol{p})\right)<0$. if

Because by Lemma $5.1 \mathfrak{f}$ is monotonically increasing, $\mathfrak{f}(\lambda)$ has a zero in $\mathbb{T}_{-}$if and only

$$
f(-1) \leq 0 \quad \text { and } \quad \lim _{\lambda \uparrow a_{\Phi}(\boldsymbol{p})-\lambda(\boldsymbol{q})} \mathfrak{f}(\lambda)>0
$$

Similarly, $\mathfrak{f}(\lambda)$ has a zero in $\mathbb{T}_{+}$if and only if

$$
f(1) \geq 0 \quad \text { and } \quad \lim _{\lambda \downarrow a_{\Phi}(\boldsymbol{p})+\lambda(\boldsymbol{q})} \mathfrak{f}(\lambda)<0 .
$$

Proof of Theorem 3.5. Let $\lambda \in \mathbb{T}_{-}$. By Lemma 5.2, (L) holds if

$$
\left\{\begin{array}{l}
-1-a_{\Omega}(\boldsymbol{p})+\frac{a_{\Phi}(\boldsymbol{p})+1}{\left(a_{\Phi}(\boldsymbol{p})+1\right)^{2}-\lambda(\boldsymbol{q})^{2}}\left\|\varphi_{\boldsymbol{q}}\right\|^{2} \leq 0 \\
0<a_{\Phi}(\boldsymbol{p})-\lambda(\boldsymbol{q})-a_{\Omega}(\boldsymbol{p})+\frac{\left\|\varphi_{\boldsymbol{q}}\right\|^{2}}{\lambda(\boldsymbol{q})}
\end{array}\right.
$$

which is equivalent to (3.8). Thus, (3.8) concludes (L). This proves (1) of Theorem 3.5 . The same proof works for (2).

\section{Acknowledgments}

This work was supported by JSPS Grant in Aid for Young Scientists (B) 26800054.

\section{References}

[1] Aharonov, Y., Davidovich, L., Zagury, N.: Quantum random walks, Phy. Rev. A 48, 1687-1690 (1993)

[2] Ambainis, A., Bach, E., Nayak, A., Vishwanath, A., Watrous, J.: One-dimensional quantum walks, ACM Symp. Theor. Comupt., 37-49 (2001)

[3] Aharonov, D., Ambainis, A., Kempe, J., and Vazirani, U.: Quantum walks on graphs, Proceedings of STOC01, pp. 50-59. quant-ph/0012090.

[4] Ambainis, A.: Quantum walks and their algorithmic applications, Int. J. Quantum Inf. 1, 507-518, (2003) 
[5] Ambainis, A., Kempe, J., Rivosh, A.: Coins make quantum walks faster, ACMSIAM Symp. Discrete Algorithm, 1099-1108 (2005)

[6] Bach, V., Fröhlich, J., Sigal, I. M.: Renormalization group analysis of spectral problems in quantum field theory, Adv. in Math., 137, 205, (1998).

[7] Cantero, M. J., Grünbaum, F. A., Moral, L., Velázquez, L.: One dimensional quantum walks with one defect, Rev. Math. Phys. 24, 1250002 (2012).

[8] Childs, A. M., Farhi, E., Gutmann, S.: An example of the difference between quantum and classical random walks, Quantum Inf. Process. 1, 35-43 (2002).

[9] Feshbach, H.: Unified theory of nuclear reactions, Ann. Phys. 5, 357-390 (1958).

[10] Di Franco, C., Mc Gettrick, M., Busch, Th.: Mimicking the probability distribution of a two-dimensional Grover walk with a single-qubit coin. Phys. Rev. Lett. 106, $080502(2011)$.

[11] Fuda, T., Funakawa, D., Suzuki, A.: Weak limit theorem for a one-dimensional split-step quantum walk, in preparation.

[12] Fuda, T., Funakawa, D., Suzuki, A.: Localization of a one-dimensional split-step quantum walk with a position-dependent coin, in preparation.

[13] Gudder, S.: Quantum Probability, Academic Press Inc., Boston (1988).

[14] Grover, L.: A fast quantum mechanical algorithm for database search, ACM Symp. Theor. Comp., 212-219 (1996).

[15] Grössing, G., Zelilinger, A.: Quantum cellular automata, Complex Systems 2, 197 - 208 (1988).

[16] Higuchi, Yu., Konno, N., Sato, I., Segawa, E. : Spectral and asymptotic properties of Grover walks on crystal lattices, J. Funct. Anal. 267, 4197-4235 (2014)

[17] Higuchi, Yu., Segawa, E.: The spreading behavior of quantum walks induced by drifted random walks on some magnifier graph, arXiv:1506.00381.

[18] Y. Higuchi, E. Segawa, A. Suzuki, Spectral mapping theorem of an abstract quantum walk, arXiv:1506.06457

[19] Hiroshima, F., Sasaki, I., Shirai, T., Suzuki, A.: Note on the spectrum of discrete Schroedinger operators, J. Math-for-Ind. 4, 105-108 (2012).

[20] Inui, K., Konishi, Y., Konno, N.: Localization of two-dimensional quantum walks, Phys. Rev. A69, 052323 (2004).

[21] Kempe, J.: Quantum random walks: An introductory overview, Contemp. Phys. 44, 307-327 (2003). 
[22] Kendon, V.: Quantum walks on general graphs, Int. J Quantum Inf. 4, 791-805 (2006).

[23] Kitagawa, T., Rudner, M. S., Berg, E., Demler, E. : Exploring topological phases with quantum walks, Phys. Rev. A, 82, 033429, (2010).

[24] Konno, N.: Quantum walks, in Quantum Potential Theory (U. Franz and M. Schurmann, Eds.), pp. 309-452, Lecture Notes in Math. 1954, Springer, 2008.

[25] Mackay, T. D., Bartlett, S. D., Stephanson, L. T., Sanders, B. C.: Quantum walks in higher dimensions, J. Phys. A: Math. Gen. 35, 2745 (2002).

[26] Matsue, K., Ogurisu, O., Segawa, E.: A note on the spectral mapping theorem of quantum walk models, arXiv:1604.00581

[27] Machida, T., Segawa, E.: Trapping and spreading properties of quantum walk in homological structure, Quantum Inf. Process. 14 (2015) 1539-1558.

[28] Meyer, D: From quantum cellular automata to quantum lattice gases, J. Stat. Phys., 85, 551-574 (1996).

[29] Magniez, F., Nayak, A., Roland, J., Santha, M.: Search via quantum walk, ACM Symp. Theor. Comput., 575-584 (2007)

[30] Magniez, F., Nayak, A., Richter, P., Santha, M.: On the hitting times of quantum versus random walks, Algorithmica, Springer (2009).

[31] H. Ohno, Unitary equivalent classes of one-dimensional quantum walks, Quantum Inf. Process., 15, 3599, (2016).

[32] Szegedy, M.: Quantum speed-up of Markov chain based algorithms, Ann. IEEE. Symp. Found., 32-41 (2004)

[33] Segawa, E.: Localization of quantum walks induced by recurrence properties of random walks, J. Comput. Theor. Nanos. 10, 1583-1590 (2013).

[34] E. Segawa, A. Suzuki, Generator of an abstract quantum walk, Quantum Stud.: Math. Found., 3, 11, (2016).

[35] Shenvi, N., Kempe, J., Whaley, K.: Quantum random-walk search algorithm, Phys. Rev. A 67, 052307 (2003)

[36] Tregenna, B., Flanagan, W., Maile, W., Kendon, V.: New J. Phys. 5, 83 (2003).

[37] Venegas-Andraca, S. E.: Quantum walks: a comprehensive review, Quantum Inf. Process. 11, 1015-1106 (2012).

[38] Watrous, J.: Quantum simulations of classical random walks and undirected graph connectivity, J. Comput. Syst. Sci. 62, 376-391 (2001) 